\title{
1. \\ Über die Reduction dreifacher Integrale auf Quadraturen.
}

(Von Dr. A. Winckler, Professor der Geodäsie an der K. K. technischen Lehr-Anstalt zu Brünn.)

$\mathbf{D}_{\text {as hier Folgende enthält die Entwickelung von Reductionsformeln }}$ für dreifache Integrale, bei welchen die Function unter den Integralzeichen nur durch ihr Argument näher bestimmt ist, und wobei die untern Grenzen sämmtlich Null, die obern willkurliche Functionen sind. Es wird dabei nur vorausgesetzt, die obern Grenzen seien von der Beschaffenheit, dafs alle später vorkommenden Gleichungen, welche sich auf die Bestimmung der Grenzen beziehen, innerhalb der in Frage kommenden Intervalle, jedesmal nur eine einzige reelle Wurzel haben und die Betrachtung es nur mit einförmigen, ohne Wechsel entweder wachsenden oder abnehmenden Functionen, oder mit stets auf- oder absteigenden Curvenzweigen zu thun habe.

Es sind dies dieselben Voraussetzungen, welche den Resultaten zu Grunde liegen, die ich im 45ten Bande dieses Journals entwickelt habe. Im Allgemeinen läfst sich bekanntlich jedes dreifache Integral so zerlegen, dafs seine einzelnen Bestandtheile in der That jenen Voraussetzungen entsprechen.

Obgleich nun die folgenden Ergebnisse auch aus den analogen Formeln für doppelte Integrale gefunden werden können, so führt doch der directe Weg schneller zum Ziele; wobei noch ein Mittel zur Prüfung übrig bleibt. Unter dem gegebenen dreifuchen Inleyrale stellen wir uns, wie üblich, den Ausdruck einer Masse vor, welche, in einem durch die Integrations-Grenzen bestimmten Raume enthalten und nur nach gewissen Schichten, deren Lage und Gestalt das Argument der Function angiebt, von unveründerlicher Dichtigkeit ist.

1.

Bezeichnet $t$ dieses Argument, d. h. die Verbindung, in welcher die Variabeln $x, y, z$ in der Function unter den Integralzeichen ansschliefslich auftreten, so wird sich der Zusammenhang aller, einem constanten $t$ entsprechenden Werthe von $x, y, z$ durch eine krumine Fläche vorstellig machen 
lassen, für welche $f(t)$, also die Dichtigkeit der Masse, unverïnderlich bleibt. Läfst man nun $t$ um $d t$ sich ändern, so wird dem Werthe $t+d t$ eine zweite krumme Fläche derselben Art entsprechen, welche mit der erstern eine räumliche Schicht einschliefst, von welcher ein Element als schiefwinkliges Parallelepiped betrachtet wird, dessen auf einander senkrechte Dimensionen $d x, d y,\left(\frac{d z}{d t}\right) d t$ sind, dessen Inhalt also

$$
=\left(\frac{d z}{d t}\right) d t d x d y
$$

ist. Der Inhalt $\boldsymbol{d} \mathbf{S}$ der bis zu den äufseren Begrenzungen des ganzen Raums sich erstreckenden Schicht wird man durch Integralion nach $x$ und $y$ zwischen den gehörigen Grenzen, also aus der Gleichung

$$
d S=d t \int d x \int\left(\frac{d z}{d t}\right) d y
$$

finden. Integrirt man endlich $f(t) d S$ nach $t$, wodurch man die Summe der in den auf einanderfolgenden Schichten enthaltenen Massen erhält, so ergiebt sich für den Ausdruck der Masse eines Theils des Ganzen:

$$
=\int f(t) d t \int d x \int\left(\frac{d z}{d t}\right) d y \text {. }
$$

Die Werthe von $x$ und $y$, zwischen welchen $f(t) d S$ zu integriren ist, um den einer gegebenen Schicht entsprechenden Werth zu finden, müssen aus dem Umfange der Projection jener Schicht (oder vielmehr der krummen Fläche, in welcher sie liegt,) auf die Ebene der $x y$, abgeleitet werden. Das Nähere hierüber wird sich am besten bei der Behandlung einzelner Fälle zeigen lassen. Wir werden dabei immer beobachten, dafs zuerst nach $y$, dann nach $x$ und schliefslich nach $t$ integrirt wird.

Offenbar ist es aber im Allgemeinen hinsichtlich des Resultats gleichgültig, ob man die in einer Schicht enthaltene Masse aus deren Projection auf die Ebene der $x \approx$, oder $y z$, oder auf irgend eine andere Fläche, oder, wie oben angenommen, aus der Projection auf $x y$ bestimmt.

Aus dieser Bemerkung ergiebt sich jedoch für doppelte Integrale eine Transformations-Gleichung, welche wohl zu beachten ist, und welche wir daher vor Allem hier anführen wollen.

Man stelle sich nämlich vor, es handle sich um die Bestimmung der Masse einer unendlich dünnen Schicht (Fig. 1.), zwischen zwei aufeinander- 
1. Winckler, über die Reduction dreifacher Integrale auf Quadraturen.

folgenden Flächen enthalten, deren Gleichung:

$$
z=\pi(x, y, t)
$$

ist, und welche von den Seitenflächen eines Parallelepipeds $(\xi, \eta, \zeta)$ begrenzt wird. Aus der eben angeführten Gleichung leite man

$$
\boldsymbol{x}=\psi(\boldsymbol{y}, \boldsymbol{z}, \boldsymbol{t}), \quad \boldsymbol{y}=\varphi(\boldsymbol{x}, \boldsymbol{z}, \boldsymbol{t})
$$

ab, und stelle sich unter $F(x, y, \pi(x, y, t))$ die Dichtigkeit der Masse im Puncte $x, y, z$ der dem Parameter $t$ entsprechenden Schicht vor, so wird man, der obigen Bemerkung gemäfs, zu den folgenden Gleichungen gelangen:

$$
\text { (A.) } \begin{aligned}
& \int_{\eta}^{\varphi(\xi, \zeta, t)} d y \int_{\xi}^{\psi(y, \zeta, t)} \boldsymbol{F}(x, y, \pi)\left(\frac{d \pi}{d t}\right) d x \\
= & \int_{\xi}^{\pi(\xi, \eta, t)} d z \int_{\eta}^{\varphi(\xi, z, t)} \boldsymbol{F}(\psi, y, z)\left(\frac{d \psi}{d t}\right) d y \\
= & \int_{\xi}^{\psi(\eta, \zeta, t)} d x \int_{\xi}^{\pi(x, \eta, t)} \boldsymbol{F}(x, \varphi, z)\left(\frac{d \varphi}{d t}\right) d z .
\end{aligned}
$$

Im Nachstehenden findet diese Gleichung, wo es sich um die Herstellung symmetrischer Formen handelt, mehrfache Anwendung.

Im Verlaufe unserer Betrachtungen ist öfters von der Auflösung gewisser Gleichungen die Rede.

Um Wiederholungen zu vermeiden, sollen durchgehends folgende Bezeichnungen gebraucht werden. Es sei

$$
t=F(x, y, z)
$$

das gegebene Argument. Vorausgesetzt wird, man habe

$$
\begin{aligned}
& y=\varphi(x, t) \text { aus der Gleichung } F[x, y, \theta(x, y)]-t=0, \\
& \boldsymbol{x}=\boldsymbol{\omega}(\boldsymbol{t}) \quad-\quad-\quad \boldsymbol{F}[x, 0, \theta(x, 0)]-t=0, \\
& \boldsymbol{x}=\lambda(\boldsymbol{t}) \quad-\quad \text { - } \quad \text { - } \boldsymbol{F}[\boldsymbol{x}, \chi(x), 0]-\boldsymbol{t}=\mathbf{0}, \\
& \boldsymbol{x}=\psi(\boldsymbol{t}) \quad-\quad-\quad-\quad \boldsymbol{F}[\boldsymbol{x}, \chi(x), \theta(x, \chi(x))]-t=0
\end{aligned}
$$

abgeleitet, wobei $\chi(x)$ und $\theta(x, y)$ die Grenzfunctionen des vorgelegten dreifachen Integrals, resp. nach $y$ und $z$ bezeichnen. Die geometrische Bedeutung dieser Gleichungen läfst sich ohne weitere Erklärung erkennen.

Es sei nun:

$$
\int_{0}^{\cdot \xi} d x \int_{0}^{\chi^{(x)}} d y \int_{y}^{\cdot \theta(x, y)} f(a x+b y+c \approx) d z
$$


4 1. Winckler, über die Reduction dreifacher Integrale anf Quadraturen.

gegeben. Alsdann ist

und man hat

$$
t=\boldsymbol{F}(x, y, z)=a x+b y+c z ;\left(\frac{d z}{d t}\right)=\frac{1}{c}
$$

$$
\begin{aligned}
& y=\varphi(x, t) \text { aus der Gleichung } a x+b y+c \theta(x, y)-t=0, \\
& x=\omega(t) \quad-\quad-\quad a x+c \theta(x, 0)-t=0 \text {, } \\
& x=\lambda(t) \quad-\quad-\quad \boldsymbol{a} x+b \chi(x)-t=0 \text {, } \\
& x=\psi(t) \quad-\quad-\quad a x+b \chi(x)+c \theta(x, \chi(x))-t=0
\end{aligned}
$$

zu berechnen.

Für die auf einander folgenden Werthe von $t$ (Fig. 2.) entsteht eine Reihe paralleler Ebenen, zwischen welchen die Dichtigkeit jedesmal consıınt ist. Um die Bestandtheile zu bezeichnen, aus welchen sich der Raum am einfachsten zusammensetzen läfst, wollen wir von jenen Ebenen diejenigen hervorheben, welche durch die sechs Puncte

$$
\begin{array}{ccc}
\{\xi, \chi(\xi), 0\}, & \{0,0,0\}, & \{0, \chi(0), 0\}, \\
\{\xi, 0, \chi(\xi, 0)\}, & \{\xi, \chi(\xi), \theta(\xi, \chi(\xi))\}, & \{0, \chi(0), \theta(0, \chi(0))\}
\end{array}
$$

gehen, und durch welche der Raum in sieben, einzeln zu berechnende Theile zerlegt wird. Drei derselben sind von der Fläche $z=\theta(x, y)$, die übrigen von Ebenen und einer cylindrischen Fläche begrenzt.

Zur Abkürzung werde, wie in allen späteren Fällen:

$$
\begin{aligned}
& \chi(\xi)=\eta, \quad \chi(0)=\eta^{\prime}, \\
& \theta(\xi, \chi(\xi))=\zeta, \quad \theta(0,0)=\zeta^{\prime \prime}, \quad \theta(0, \chi(0))=\zeta^{\prime}, \quad \theta(\xi, 0)=\zeta^{\prime \prime}
\end{aligned}
$$

gesetzt. Dann mufs man, um die einzelnen Theile des dreifachen Integrals zu finden, die Integration wie folgt sich erstrecken lassen:

(1.) Nach $y$, von 0 bis $\frac{t \text {-ax }}{b}$; Nach $x$, von $\frac{t}{a}$ bis $\xi$; Nach $t$, von $a \xi$ bis $a \xi+b \eta$;
(2.)

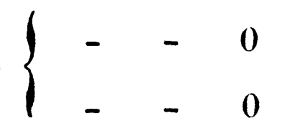
$-\frac{t-a x}{b} ; \quad-$
$-\chi(x) ; \quad-$
$\left.\begin{array}{c}-\frac{t}{a}-\lambda(t) \\ -\lambda(t)-\xi\end{array}\right\}$
$a \xi+b \eta \quad 0$
(3.)
$\begin{cases}- & -0 \\ - & -\end{cases}$
$-\frac{\boldsymbol{t}-\boldsymbol{a} x}{b}$
$\left.\begin{array}{l}-\quad 0-\lambda(t) \\ -\lambda(t)-\xi\end{array}\right\}$
(4.)
$-\chi(x)$
$-0-\xi$
$-b \eta^{\prime}$
(5.)

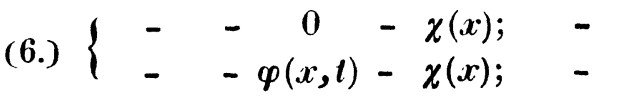
(7.)
$-\chi(x)$
$\left.\begin{array}{c}-0-\omega(t) \\ -\omega(t)-\xi\end{array}\right\}$
$b \eta^{\prime} \quad-a \xi+c \zeta^{\prime \prime} ;$
$\begin{array}{ccc}0 & -\chi(x) ; & - \\ -\varphi(x, t) & -\chi(x) ; & -\end{array}$
$-0-\omega(t)$
- $a \xi+c \zeta^{\prime \prime}-a \xi+b \eta+c \xi$
- $\quad 0-\omega(t)\}$
- $a \xi+b \eta+c \zeta-$
$b \eta^{\prime}+c \zeta^{\prime}$
- $0-\omega(t)$
- $\quad b \eta^{\prime}+c \xi^{\prime}$ $c \zeta^{0}$; 
Dieses System von Werthen läfst sich auch leicht in ein kürzeres verwandeln, wenn man es so einrichtet, dafs bei jedem einzelnen Integral die untere Grenze in Bezug auf $t=$ Null wird. Während jedoch das angegebene System nur wirkliche Bestandtheile des dreifachen Integrals enthält, kommen bei dem transformirten Integral fremde Raumtheile in Rechnung, welche sich zwar wieder aufheben, bei welchen aber möglicherweise die Eingangs gemachte Voraussetzung nicht Statt finden könnte. Das transformirte System ist:

Nach $y$, von 0 bis $\frac{t-a x}{b}$; Nach $x$, von $\xi$ bis $\frac{t}{a}$; Nach $t$, von 0 bis $a \xi$;

$$
\begin{aligned}
& \text { - } \quad \chi(x)-\frac{t-a x}{b} ; \quad-\quad-0-\lambda(t) ; \quad-\quad-0-b \eta^{\prime} ; \\
& \text { - } \quad 0 \quad-\varphi(x, t) ; \quad-\quad-0 \quad-\omega(t) ; \quad-\quad-0-c \zeta^{5} \text {; } \\
& \text { - }-\frac{t-a x}{b}-\chi(x) ; \quad-\quad-\xi-\lambda(t) ; \quad-\quad-0-a \xi+b \eta \text {; } \\
& \text { - } \quad \text { - } 0-\varphi(x, t) ; \quad-\quad-\omega(t)-\xi ; \quad-\quad-0-a \xi+c \zeta^{\prime \prime} ; \\
& \text { - } \quad-\varphi(x, t)-\chi(x) ; \quad-\quad-0-\psi(t) ; \quad-\quad-0-b \eta^{\prime}+c \zeta^{\prime} ; \\
& \text { - } \quad-\varphi(x, t)-\chi(x) ; \quad-\quad-\psi(t)-\xi ; \quad-\quad-0-a \xi+b \eta+c \xi \text {. }
\end{aligned}
$$

Ähnliche Transformationen lassen sich auch bei den folgenden Grenzsystemen machen; wir werden dieselben nicht besonders anführen.

Die Summe der hierdurch gegebenen Integrale stellt das vorgelegte dreifache Integral dar. Man kann also der Kürze wegen von der Aufstellung der Formel abstehen. Nur zwei besondere Fälle sind ausführlicher zu bemerken.

$1^{\circ}$. Es sei $a=0, b=0, c=1$, also $t=z$. Dann sind die homogenen Schichten-Ebenen parallel mit $x y$, und es ist

$$
\begin{aligned}
& y=\varphi(x, t) \text { durch die Gleichung } \theta(x, y)=t \text {, } \\
& \begin{array}{lllll}
x=\omega(t) & - & - & - & \theta \\
x=\psi(x, 0)=t,
\end{array}
\end{aligned}
$$

gegeben. Ermittelt man die Werthe der in der allgemeinen Formel vorkommenden unbestimmten Ausdrücke, so ergiebt sich nach einigen weiteren Verwandlungen die bemerkenswerthe Gleichung

$$
\begin{gathered}
\int_{0}^{\xi} d x \int_{0}^{\gamma \chi(x)} d y \int_{0}^{\theta(x, y)} f(z) d z \\
=\int_{0}^{\theta(0,0)} f(t) d t \int_{0}^{\omega(t)} \varphi(x, t) d x+\int_{0}^{\theta(\xi,())} f(t) d t \int_{\omega(t)}^{\xi \xi} \varphi(x, t) d x \\
+\int_{0}^{\theta\left(0, \chi^{(()))}\right.} f(t) d t \int_{0}^{\psi(t)}(\chi(x)-\varphi(x, t)) d x+\int_{0}^{\theta(\xi, \chi(\xi))} f(t) d t \int_{\psi(t)}^{\xi}(\chi(x)-\varphi(x, t)) d x,
\end{gathered}
$$


1. Winckler, über die Reduction dreifacher Integrale auf Quadraturen.

welche jedoch schon in den auf andere Art abgeleiteten Resultaten (im 45. Bd. dieses Journals) als specieller Fall enthalten ist.

$2^{\circ}$. Setzt man $\chi(x)=\eta, \theta(x, y)=\zeta$ constant, so ist

$$
\begin{aligned}
\varphi(x, t) & =\frac{1}{b}(t-a x-c \zeta) ; & \omega(t) & =\frac{t-c \zeta}{a}, \\
\lambda(t) & =\frac{t-b \eta}{a} ; & \psi(t) & =\frac{1}{a}(t-b \eta-c \zeta),
\end{aligned}
$$

und es findet sich nach einigen leichten Umformungen folgende symmetrische Gleichung :

$$
\begin{gathered}
2 a b c \int_{0}^{\zeta} d x \int_{0}^{\eta} d y \int_{0}^{\zeta} f(a x+b y+c z) d z \\
=\int_{1}^{a \xi}(t-a \xi)^{2} f(t) d t+\int_{0}^{b \eta}(t-b \eta)^{2} f(t) d t+\int_{0}^{c \zeta}(t-c \zeta)^{2} f(t) d t \\
-\left\{\int_{0}^{a \xi+b \eta}(a \xi+b \eta-t)^{2} f(t) d t+\int_{0}^{a \xi+c \zeta}(a \xi+c \zeta-t)^{2} f(t) d t\right. \\
\left.+\int_{11}^{b \eta+c^{\zeta}}(b \eta+c \zeta-t)^{2} f(t) d t\right\}+\int_{0}^{a \xi+b \eta+c \zeta}(a \xi+b \eta+c \zeta-t)^{2} f(t) d t .
\end{gathered}
$$

Für das $\boldsymbol{n}$ fache Integral einer Function mit linearem Argument würde man $2^{n}-1$ Theil-Integrale erhalten.

3.

Es sei nunmehr $t$ der Quotient zweier linearen Functionen der drei Veränderlichen und zwar

$$
t=\frac{a x+b y+c z+m}{\alpha x+\beta y+\gamma z+\mu}
$$

Die Schichten von constanter Dichtigkeit sind hier gleichfalls Ebenen (Fig. 2.); ihre gemeinsame Gleichung ist

$$
(\alpha t-a) x+(\beta t-b) y+(\gamma t-c) z+\mu t-m=0
$$

und sie haben eine gemeinschaftliche Durchschnittslinie, deren Gleichungen sich ergeben, wenn man $t$ unbestimmt werden läfst. Diese Gleichungen sind also

$$
\begin{aligned}
& a x+b y+c z+m=0, \\
& \alpha x+\beta y+\gamma z+\mu=0 ;
\end{aligned}
$$

woraus sich die Coordinalen der Durchschnittspuncte dieser Geraden und der Coordinaten-Ebenen finden lassen. Um rücksichtlich der Lage dieser Geraden auf einen ganz bestimmten Fall sich beziehen zu können, mögen sämmtliche 
1. Winckler, über dic Reduction dreifacher Integrale auf Quadraturen. 7

Coëfficienten als posiliv betrachtet und es mag aufserdem angenommen werden, dafs

$$
\frac{a}{\alpha}>\frac{b}{\beta}>\frac{c}{\gamma}>\frac{m}{\mu}
$$

sei. Dann liegt die Durchschnittslinie aller Ebenen unterhalb des positiven Theils der $x y$ Ebene und trifft dieselbe in dem Theile $(+x,-y)$. Ferner entspricht die Ehene, welche durch den Anfangspunct der Coordinaten geht, vermöge der Annahmen über die Coëfficienten, dem kleinsten Werthe von $t$, welcher in Betracht kommt. Läfst man also von dieser Lage aus die Ebene durch den auf der Seite der positiven Coordinaten liegenden Raum sich bewegen, so nimmt $t$ fortwährend zu.

Dies vorausgesetzt, haben die Grenzen, zwischen welchen die Integration für die einzelnen Theile des Volumens sich zu erstrecken hat, die in dem folgenden Schema angegebenen Werthe:

Nach $y$, von $\quad 0 \quad$ bis $-\frac{(\alpha t-a) x+\mu t-m}{\beta t-b}$; Nach $x$, von $\xi$ bis $-\frac{\mu t-m}{\alpha t-a}$;

Nach $t$, von $\frac{m}{\mu}$ bis $\frac{\alpha \xi+m}{\alpha \xi+\mu}$;

Nach $y$, von$$
\text { bis }-\frac{(\alpha t-a) x+\mu t-m}{\beta t-b} \text {; Nach } x, \text { von } 0 \quad \text { bis } \lambda(t)
$$

Nach $t$, von $\frac{m}{\mu}$ bis $\frac{b \eta^{\prime}+m}{\beta \eta^{\prime}+\mu} ;$

Nach $y$, von

0

bis $\varphi(x, t)$;

Nach $x$, von $\quad 0 \quad$ bis $\omega(t)$

Nach $t$, von $\frac{m}{\mu}$ bis $\frac{c \zeta^{0}+m}{\gamma \zeta^{0}+\mu}$

Nach $y$, von $-\frac{(\alpha t-a) x+\mu t-m}{\beta t-b}$ bis $\chi(x) ;$

Nach $x$, von $\xi \quad$ bis $\lambda(t)$;

Nach $t$, von $\frac{m}{\mu}$ bis $\frac{a \xi+b \eta+m}{\alpha \xi+\beta \eta+\mu}$;

Nach $y$, von $\varphi(x, t) \quad$ bis 0 ;

Nach $x$, von $\xi \quad$ bis $\omega(t)$;

Nach $t, \operatorname{von} \frac{m}{\mu}$ bis $\frac{a \xi+c \xi^{\prime \prime}+m}{\alpha \xi+\gamma \xi^{4 \prime}+\mu} ;$

Nach $y$, von

$$
\begin{aligned}
& \varphi(x, t) \quad \text { bis } \chi(x) ; \\
& \text { Nach } t, \text { von } \frac{m}{\mu} \text { bis } \frac{b \eta^{\prime}+c \zeta^{\prime}+m}{\beta \eta^{\prime}+\gamma \zeta^{\prime}+\mu} ;
\end{aligned}
$$

Nach $x$, von 0 bis $\psi(t)$

Nach $y$, von

$\varphi(x, t) \quad$ bis $\chi(x)$;

Nach $x$, von $\psi(t)$ bis $\xi$;

Nach $t, \operatorname{von} \frac{m}{\mu}$ bis $\frac{a \xi+b \eta+c \zeta+m}{\alpha \xi+\beta \eta+\gamma \zeta+\mu}$. 
8 1. Wincliler, über die Reduction dreifacher Integrale auf Quadraturen.

Noch ist zu bemerken, dafs

$$
\left(\frac{d z}{d t}\right)=\frac{(\alpha c-a \gamma) x+(\beta c-b \gamma) y+\mu c-m \gamma}{(\gamma t-c)^{2}}
$$

ist, und dafs dieser Ausdruck für alle in Betracht kommenden Werthe von $t$ stets positiv bleibt. Hiermit ist das dreifache Integral als vollständig gefunden zu betrachten. Der grofsen Weitläuftigkeit wegen, welche die weitere Ausführung haben würde, möge es bei diesen Angaben bewenden. Es ist leicht \%u sehen, dafs hierdurch, selbst wenn $\%(x)$ und $\theta(x, y)$ nicht näher bestimmt sind, das gegebene dreifache Integral durch eine Summe einfacher und doppelter Integrale ausgedrückt wird.

4.

Nur der besondere Fall mag näher erörtert werden, wenn die obern Grenzen $\chi(x)=\eta, \theta(x, y)=\zeta$ constunt sind. Alsdann ist

$$
\begin{array}{rlrl}
\varphi(x, t) & =-\frac{(\alpha t-a) x+(\gamma t-r) \zeta+\mu t-m}{\beta t-b}, & \omega(t)=-\frac{(\gamma t-c) \zeta+\mu t-m}{\alpha t-a}, \\
\lambda(t)=-\frac{(\beta t-b) \eta+\mu t-m}{\alpha t-a}, & \psi(t)=-\frac{(\beta t-b) \eta+(\gamma t-c) \zeta+\mu t-m .}{\alpha t-a} .
\end{array}
$$

Selzt man der Kürze wegen:

$$
\begin{aligned}
\alpha m-a \mu=a_{1}, & \beta m-b \mu=b_{1}, \quad \gamma m-c \mu=c_{1}, \\
\beta c-b \gamma=\alpha_{1}, & \gamma a-c \alpha=\beta_{1}, \quad \alpha b-a \beta=\gamma_{1},
\end{aligned}
$$

und bemerkt, dafs die Summe der sieben einzelnen Integrale eine kürzere Form annimmt, wenn man dieselben in zwölf andere Integrale zerlegt, von welchen drei. mit $\frac{m}{\mu}$ und die übrigen in symmetrischer Weise mit den zwischen dem ersten und letzten der im obigen Schema vorkommenden Grenzausdrücke ihren Anfang nehmen, so findet man:

$$
\begin{aligned}
& \text { 1.2.3. } \alpha_{1} \beta_{1} \gamma_{1} \int_{1}^{\xi} d x \int_{0}^{\eta} d y \int_{11}^{\zeta} f\left(\frac{a x+b y+c z+m}{\alpha x+\beta y+\gamma z+\mu}\right) d z
\end{aligned}
$$

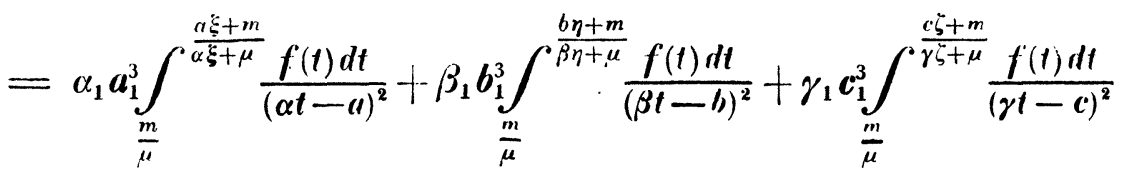

$$
\begin{aligned}
& +\alpha_{1}\left(\beta_{1} \zeta-a_{1}\right)^{3} \int_{\frac{c \zeta+m}{\gamma \zeta+\mu}}^{\frac{a \xi+c ;+m}{\alpha \xi+\gamma \zeta+\mu}} \frac{f(t) d t}{(\alpha t-a)^{2}}+\beta_{1}\left(\gamma_{1} \xi-b_{1}\right)^{3} \int_{\frac{a \xi+m}{\alpha \xi+\mu}}^{\frac{a \xi+b \eta+m}{\alpha \xi+\beta \eta+\mu}} \frac{f(t) d t}{(\beta t-b)^{2}} \\
& +\gamma_{1}\left(\alpha_{1} \eta-c_{1}\right)^{3} \int_{\frac{b \eta+m}{\beta \eta+\mu}}^{\frac{b \eta+c \zeta+m}{\beta \eta+\gamma \zeta+\mu}} \frac{f(t) d t}{(\gamma t-c)^{2}}
\end{aligned}
$$


1. Winckler, über die Reduction drcifacher Integrale auf Quadraturen.

$$
\begin{aligned}
& -\alpha_{1}\left(\gamma_{1} \eta+a_{1}\right)^{3} \int_{\frac{b \eta+m}{\beta \eta+\mu}}^{\frac{a \xi+b \eta+m}{\alpha \xi+\beta \eta+\mu}} \frac{f(t) d t}{(\alpha t-a)^{2}}-\beta_{1}\left(\alpha_{1} \zeta+b_{1}\right)^{3} \int_{\frac{c \zeta+m}{\gamma \zeta+\mu}}^{\frac{b \eta+c \zeta+m}{\beta \eta+\gamma \zeta+\mu}} \frac{f(t) d t}{\beta(t-b)^{2}} \\
& -\gamma_{1}\left(\beta_{1} \xi+c_{1}\right)^{3} \int_{\frac{a \xi+m}{\alpha \xi+\mu}}^{\frac{a \xi+c \zeta+m}{\alpha \xi+\gamma \zeta+\mu}} \frac{f(t) d t}{(\gamma t-c)^{2}} \\
& +\alpha_{1}\left(\gamma_{1} \eta-\beta_{1} \zeta+a_{1}\right)^{3} \int_{\frac{b \eta+c \zeta+m}{\beta \eta+\gamma \zeta+\mu}}^{\frac{a \xi+b \eta+c \zeta+m}{\alpha \xi+\beta \eta+\gamma \zeta+\mu}} \frac{f(t) d t}{(\alpha t-a)^{2}}+\beta_{1}\left(\alpha_{1} \zeta-\gamma_{1} \xi+b_{1}\right)^{3} \int_{\frac{a \xi+c \zeta+m}{\alpha \xi+\gamma \zeta+\mu}}^{\frac{a \xi+b \eta+c \zeta+m}{\alpha \xi+\beta \eta+\gamma \zeta+\mu}} \frac{f(t) d t}{(\beta t-b)^{2}} \\
& +\gamma_{1}\left(\beta_{1} \xi-\alpha_{1} \eta+c_{1}\right)^{3} \int_{\frac{a \xi+b \eta+m}{\alpha \xi+\beta \eta+\mu}}^{\frac{a \xi+b \eta+c \zeta+m}{\alpha \xi+\beta \eta+\gamma \zeta+\mu}} \frac{f(t) d t}{(\gamma t-c)^{2}} .
\end{aligned}
$$

In dieser, wie man sieht, ganz symmetrischen Formel sind viele specielle Resultate enthalten.

Schliefslich die Bemerkung, dafs sich aus den bisherigen, so wie auch aus den noch folgenden Resultaten, Reductionsformeln für 4, 5, . -fache Integrale finden lassen, zu deren Ermittelung sich das geometrische Verfahren direct nicht mehr benutzen läfst. In dem eben betrachteten Falle z. B. braucht man nur eine weitere Veränderliche $s$ einzuführen, indem man $m s+n$ statt $m$ und $\mu s+\nu$ statt $\mu$ setzt, die Gröfse $s$ sich ebenfalls in den Grenzen enthalten vorstellt, so dafs diese nun $\chi(x, s), \theta(x, y, s)$ sind, endlich aber $\pi(s)$ statt $\xi$ setzt. Integrirt man hierauf die für das dreifache Integral gefundene Gleichung weiter nach $s$, von 0 bis $\sigma$, so wird man eine Gleichung für das vierfache Integral

$$
\int_{0}^{\sigma} d s \int_{0}^{\pi(s)} d x \int_{0}^{\chi^{(x, s)}} d y \int_{0}^{\theta(x, y, s)} f\left(\frac{a x+b y+c z+m s+n}{\alpha x+\beta y+\gamma z+\mu s+\nu}\right) \cdot d z
$$

finden, die durch doppelte und dreifache, nach $1, s$ und nach $t, s, x$ genommene Integrale, ausgedrückt ist. Ähnlich wäre der Weg, um aus den Reductionsformeln für doppelte, diejenigen für dreifache Integrale abzuleiten.

5.

Es sei nun $t$ eine ganze rationale Function zweiten Grades und zwar:

$$
t=a x^{2}+b y^{2}+c z^{2}+2 h x y+2 k x z+2 l y z+2 m x+2 n y+2 r z .
$$

Zur Vereinfachung nehme man an, die drei letzten Glieder dieses Ausdruckes seien dadurch weggeschafft worden, dafs man $x+x_{0}, y+y_{0}, z+z_{0}$ Crelle's Journal f. d. M. Bd. L. Heft 1. 
10 1. Winckler, über die Reduction dreifacher Integrale anf Quadraturen.

statt $x, y, z$ gesetzt und dann $x_{10}, y_{0}, z_{0}$ der Forderung gemäfs bestimmt habe. Dies kann bekanntlich nur in dem einzigen Falle nicht geschehen, wenn $x_{0}, y_{0}, z_{0}$ unendlich werden, also der Ausdruck

$$
\Delta=a l^{2}+b k^{2}+c h^{2}-a b c-2 h k l
$$

verschwindet. Fände dieser Fall, welcher jedoch ausgeschlossen bleiben mag, Statt, so liefse sich das entsprechende Integral ähnlich wie in den folgenden Fällen behandeln.

Es sei also

$$
t=a x^{2}+b y^{2}+c z^{2}+2 h x y+2 k x z+2 l y z,
$$

so sind die dieser Gleichung zugehörenden Flächen, je nach Beschaffenheit der Coëfficienten, entweder Ellipsoüde oder Hyperboloüde, deren Mittelpuncte mit dem Ursprunge der Coordinaten zusammenfallen.

Zunächst für den ersten Fall mögen die Coëfficienten insgesammt als positiv betrachtet werden. Nimmt man aufserdem an, es sei

$$
a b>h^{2}, \quad a c>k^{2}, \quad b c>l^{2},
$$

so ist die krumme Fläche (Fig. 3.) das dreiaxige Ellipsoüd, welches zu den Coordinaten-Ebenen eine solche Lage hat, dafs der zur $z, y, x-$ Axe conjugirte Durchmesser des Hauptschnitts resp. in der $x y, z x, y z-E b e n e$ zwischen den Halb-Axen $(f-x,-y),(f z,-x),(+y,-z)$ hindurchgeht. Diese Angaben sind zur Bestimmung der Grenzen hinreichend. Das entsprechende dreifache Integral zerfällt auch hier in sieben einzelne Integrale, welche sich auf ganz ähnliche Weise wie in dem vorigen Falle finden lassen. Auch ist leicht zu bemerken, dafs die Theilung des Raums ganz ähnlich wie im vorigen Falle geschehen kann, weshalb nur noch das System der Grenzwerthe wie folgt anzugeben ist. Es ist zu integriren:

Nach $y$, von $0 \quad$ bis $-\frac{h x-\sqrt{ }\left(b t+\left(h^{2}-a b\right) x^{2}\right)}{b} ;$ Nach $x$, von 0 bis $/ \frac{t}{a}$;

$$
\text { Nach } t \text {, von } \quad 0 \text { bis } a \xi^{2} \text {; }
$$

Nach $y$, von $\quad 0 \quad$ bis $-\frac{h x-\sqrt{ }\left(b t+\left(h^{2}-a b\right) x^{2}\right)}{6} ;$ Nach $x$, von 0 bis $\xi$;

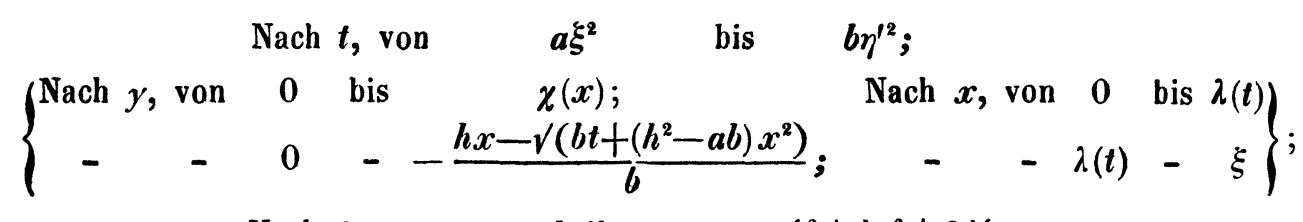

$$
\text { Nach } t \text {, von } \quad b \eta^{\prime 2} \quad \text { bis } a \xi^{2}+6 \eta^{2}+2 h \xi \eta \text {; }
$$


1. Winclkler, ̈̈ber die Reduction dreifacher Integrale auf Quadraturen.

Nach $y$, von 0 bis $\quad \chi(x)$; $\quad$ Nach $x$, von 0 bis $\xi$; Nach $t$, von $a \xi^{2}+b \eta^{2}+2 h \xi \eta$ bis $c \xi^{0^{2}}$;

(Nach $y$, von $\varphi(x, t)$ bis $\quad \chi(x)$;

Nach $x$, von 0 bis $\omega(t)\}$.

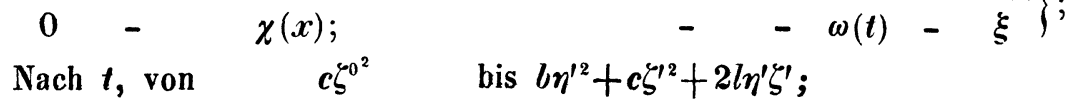

\{ Nach $y$, von $\varphi(x, t)$ bis $\quad \chi(x)$;

Nach $x$, von $\psi(t)$ bis $\omega(t)$

Nach $t$, von $b \eta^{\prime 2}+c \zeta^{\prime 2}+2 l \eta^{\prime} \zeta^{\prime}$ bis $a \xi^{2}+c \zeta^{\prime \prime 2}+2 k \xi \xi^{\prime \prime}$;

Nach $y$, von $\varphi(x, t)$ bis $\quad \chi(x) ; \quad$ Nach $x$, von $\psi(t)$ bis $\quad \xi$;

Da ferner :

$$
\text { Nach } t \text {, von } a \xi^{2}+c \xi^{\prime \prime 2}+2 k \xi \zeta^{\prime \prime} \text { bis }\left\{\begin{array}{c}
a \xi^{2}+b \eta^{2}+c \zeta^{2} \\
+2 h \xi \eta+2 k \xi \xi+2 l \eta \zeta .
\end{array}\right.
$$

$$
\left(\frac{d z}{d t}\right)=\frac{1}{2 \sqrt{ }\left(c t-\left(a c-k^{2}\right) x^{2}-2(h c-k l) x y-\left(b c-l^{2}\right) y^{2}\right)}
$$

ist, so folgt

$$
d S=\frac{d t}{2 \sqrt{ }\left(b c-l^{2}\right)} \int\left(\arcsin \frac{(h c-k l) x+\left(b c-l^{2}\right) y}{\sqrt{c} \sqrt{\left(\Delta x^{2}+\left(b c-l^{2}\right) t\right)}}+\text { Const. }\right) d x .
$$

Statt der weitern Ausführung möge der specielle Fall betrachtet werden, in welchem die Grenzen des dreifachen Integrals constant und $h=k=l=0$ sind. Dann ist

$$
\begin{aligned}
\varphi(x, t) & =\sqrt{ }\left(\frac{t-c \zeta^{2}-a x^{2}}{b}\right), & \omega(t) & =\sqrt{ }\left(\frac{t-c \zeta^{2}}{a}\right), \\
\lambda(t) & =\sqrt{ }\left(\frac{t-b \eta^{2}}{a}\right), & \psi(t) & =\sqrt{ }\left(\frac{t-b \eta^{2}-c \zeta^{2}}{a}\right) .
\end{aligned}
$$

Bemerkt man aufserdem, dafs für ein negatives $\alpha$,

$$
\begin{aligned}
& \int \arcsin \frac{\gamma}{\sqrt{\left(\alpha x^{2}+\beta\right)}} d x=x \arcsin \frac{\gamma}{\sqrt{\left(\alpha x^{2}+\beta\right)}}+\frac{\gamma}{\sqrt{-\alpha}} \arcsin \frac{x \sqrt{ }-\alpha}{\sqrt{\left(\beta-\gamma^{2}\right)}} \\
& -\sqrt{(}\left(-\frac{\beta}{\alpha}\right) \arcsin \frac{\gamma \sqrt{ }(-\alpha)-x}{\sqrt{\left(\left(\beta-\gamma^{2}\right)\left(\alpha x^{2}+\beta\right)\right)}}+\text { Const. }
\end{aligned}
$$

ist, und formt die einzelnen sich ergebenden Integrale so um, dafs ein symmetrisches, in allen Theilen reelles Resultat entsteht, so wird sich folgende Gleichung finden:

$$
\begin{gathered}
2 \sqrt{a b c} \int_{0}^{\zeta \xi} d x \int_{0}^{\eta} d y \int_{0}^{\zeta} f\left(a x^{2}+b y^{2}+c z^{2}\right) d z \\
=\frac{1}{2} \pi\left\{\int_{a \xi^{2}}^{a \xi^{2}+b \eta^{2}+c \zeta^{2}} f(t)\left(\xi \sqrt{ } a-V^{\prime} t\right) d t+\int_{b \eta^{2}}^{a \xi^{2}+b \eta^{2}+c \xi^{2}} f(t)(\eta \sqrt{ } b-\sqrt{ } t) d t\right. \\
\left.+\int_{c \zeta^{2}}^{a \xi^{2}+b \eta^{2}+c \zeta^{2}} f(t)(\zeta \sqrt{ } c-\sqrt{ } t) d t+\int_{0}^{a \xi^{2}+b \eta^{2}+c \zeta^{2}} f(t) \sqrt{ } t . d t\right\}
\end{gathered}
$$


1. Winckler, über die Reduction dreifacher Integrale auf Quadraturen.

$$
\begin{aligned}
& -\int_{a \xi^{2}+b \eta^{2}}^{a \xi^{2}+b \eta^{2}+c \xi^{2}} f(t)\left\{\xi \sqrt{ } a \arccos \frac{\eta \sqrt{ } b}{\sqrt{\left(t-a \xi^{2}\right)}}-\sqrt{ } t \arccos \frac{\xi \eta \sqrt{ } b}{\sqrt{\left(\left(t-a \xi^{2}\right)\left(t-b \eta^{2}\right)\right)}}\right. \\
& \left.+\eta \sqrt{b} \arccos \frac{\xi \sqrt{ } a}{\sqrt{\left(t-b \eta^{2}\right)}}\right\} d t \\
& -\int_{a \xi^{2}+c \zeta^{2}}^{a \xi^{2}+b \eta^{2}+c \zeta^{2}} f(t)\left\{\zeta \sqrt{ } c \arccos \frac{\xi \sqrt{ } a}{\sqrt{\left(t-c \zeta^{2}\right)}}-\sqrt{ } t \arccos \frac{\xi \zeta \sqrt{ } a c}{\sqrt{\left(\left(t-a \xi^{2}\right)\left(t-c \zeta^{2}\right)\right)}}\right. \\
& \left.+\xi \sqrt{ } a \arccos \frac{\zeta \sqrt{ } c}{\sqrt{\left(t-a \xi^{2}\right)}}\right\} d t \\
& -\int_{b \eta^{2}+c \zeta^{2}}^{a \xi^{2}+b \eta^{3}+c \zeta^{2}} f(t)\left\{\eta \sqrt{ } b \arccos \frac{\zeta \sqrt{ } c}{\sqrt{\left(t-b \eta^{2}\right)}}-\sqrt{ } t \arccos \frac{\eta \zeta \sqrt{ } b c}{\left.\sqrt{(}\left(t-b \eta^{2}\right)\left(t-c \zeta^{2}\right)\right)}\right. \\
& \left.+\zeta \sqrt{ } \arccos \frac{\eta \sqrt{ } b}{\sqrt{\left(t-c \zeta^{2}\right)}}\right\} d t \text {. }
\end{aligned}
$$

Hieraus ergiebt sich, z. B. wenn $f(t)$ für $t=\infty$ verschwindet, die bekannte Gleichung

$$
\int_{0}^{\infty} d x \int_{0}^{\infty} d y \int_{0}^{\infty} f\left(a x^{2}+b y^{2}+c z^{2}\right) d z=\frac{\pi}{4 \sqrt{ }(a b c)} \int_{0}^{\infty} f(t) \cdot V t \cdot d t .
$$

6.

Auch wenn die Coëfficienten $a, b, c$ verschiedene Zeichen haben, bleibt das obige Verfahren afwendbar. Um hier alle Fälle zu umfassen, genügt es, anzunehmen, es sei einer dieser Coëfficienten, z. B. a, negativ und die beiden andern seien positiv. Auch werde $h, k, l$ als positiv und $l^{2}-b c$ als negativ angesehen. Dann ist in Folge dieser Annahmen

$$
h^{2}>a b, \quad k^{2}>a c, \quad l^{2}<b c
$$

und man erhält, während $t$ alle positiven und negativen Werthe durchläuft, alle ein - und zweitheiligen elliptischen Hyperbolöde, welche sich an einen elliptischen Kegel asymptotisch anschliefsen. Noch ist zu bemerken, dafs, vermöge der obigen Annahmen, die den Axen der $y$ und $z$ conjugirten Durchmesser der resp. in der $x y$ - und $x z$-Ebene vorkommenden Hyperbeln, so wie der zur $z$-Axe conjugirte Durchmesser der Ellipsen in der $y z-E b e n e$, nicht in die Räume der positiven Halb-Axen fallen.

Im Allgemeinen zerfällt das dreifache Integral auch hier in sieben Bestandtheile. Um imaginüre Ausdrücke zu vermeiden, mufs man bei der Aufstellung der Grenzen unterscheiden, ob die Asymptoten der Hyperbeln in der $x y$ - und $x z$-Ebene die Ordinaten $\chi(\xi)$ und $\theta(\xi, 0)$ selbst, oder nur deren Verlängerungen schneiden. Aus dieser Unterscheidung entspringen vier 
1. Winclkler, über die Reduction dreifacher Integrale auf Quadraturen. 13 verschiedene Fälle, welche durch die folgenden Ungleichheiten characterisirt werden :

1. $a \xi^{2}+b \chi(\xi)^{2}+2 h \xi \chi(\xi)>0$ und $a \xi^{2}+c \theta(\xi, 0)^{2}+2 k \xi \theta(\xi, 0)>0$,

2.

3.

4.
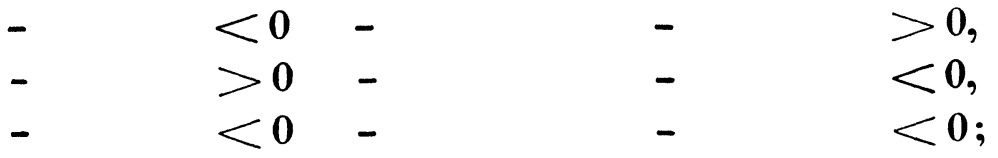

wo das Zeichen $>$ anzeigt, dafs die Asymptote die betreffende Ordinate selbst, das Zeichen $<$, dafs sie blofs deren Verlängerung schneidet.

Von diesen vier Fällen (Fig. 4.) möge z. B. der erste etwas näher betrachtet werden. Die entsprechenden Grenzwerthe sind:

Nach $y$, von $\quad 0 \quad$ bis $-\frac{h x-\sqrt{ }\left(b t+\left(h^{2}-a b\right) x^{2}\right)}{b} ;$ Nach $x$, von $\sqrt{\frac{t}{a}}$ bis $\xi$;

Nach $t$, von $a \xi^{2}$ bis 0 ;

Nach $y$, von $\quad 0 \quad$ bis $-\frac{h x-\sqrt{ }\left(b t+\left(h^{2}-a b\right) x^{2}\right)}{b}$; Nach $x$, von 0 bis $\xi$;

Nach $t$, von 0 bis $a \xi^{2}+b \eta^{2}+2 k \xi \eta$;

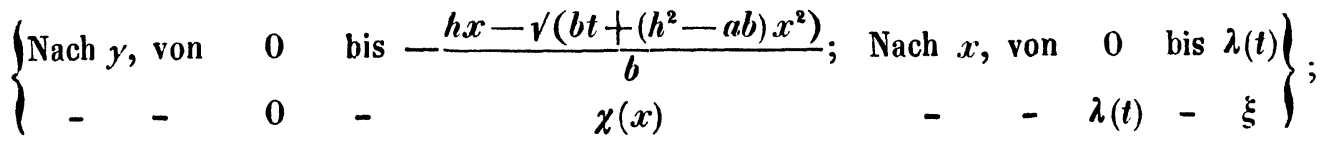

Nach $t$, von $a \xi^{2}+b \eta^{2}+2 h \xi \eta$ bis $b \eta^{\prime 2}$;

Nach $y$, von 0 bis $\quad \chi(x) ; \quad$ Nach $x$, von 0 bis $\xi$;

Nach $t$, von $b \eta^{\prime 2}$ bis $a \xi^{2}+c \zeta^{\prime \prime 2}+2 k \xi \zeta^{\prime \prime}$;

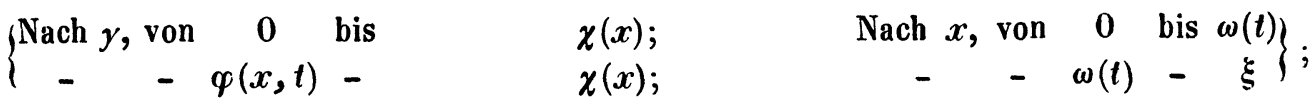

Nach $t$, von $a \xi^{2}+c \zeta^{\prime \prime 2}+2 k \xi \zeta^{\prime \prime}$ bis $a \xi^{2}+b \eta^{2}+c \zeta^{2}+2 h \xi \eta+2 k \xi \zeta+2 l \eta \zeta$

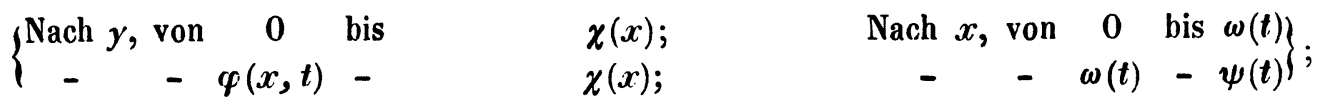

Nach $t$, von $a \xi^{2}+b \eta^{2}+c \zeta^{2}+2 h \xi \eta+2 k \xi \zeta+2 \ln \zeta$ bis $c \zeta^{0^{2}}$;

Nach $y$, von $\varphi(x, t)$ bis $\quad \chi(x) ; \quad$ Nach $x$, von 0 bis $\psi(t)$;

Nach $t$, von $c \zeta^{0^{2}}$ bis $b \eta^{\prime 2}+c \zeta^{\prime 2}+2 l \eta^{\prime} \zeta^{\prime}$.

Eine weitere Ausführung ist auch hier nicht nöthig. Als Beispiel möge jedoch der specielle Fall, in welchem die Grenzen des Integrals conslant und $h, k, l$ Null sind, vollständig erörtert werden.

Man hat

$$
d S=\frac{d t}{2 \sqrt{b c}} \int\left(\arcsin \frac{\eta \sqrt{ } b}{\sqrt{\left(t-a x^{2}\right)}}+\text { Const. }\right) d x,
$$


14 1. Winckler, über die Reduction dreifacher Integrale auf Quadraturen.

und da für ein positives $\alpha$

$$
\begin{aligned}
& \int \arcsin \frac{\gamma}{\sqrt{\left(\alpha x^{2}+\beta\right)}} d x=x \arcsin \frac{\gamma}{\sqrt{\left(\alpha x^{2}+\beta\right)}}+\frac{\gamma}{2 \sqrt{ } \alpha} \log \left(x \sqrt{ } \alpha+\sqrt{ }\left(\alpha x^{2}+\beta-\gamma^{2}\right)\right)^{2} \\
& -\frac{1}{4} \sqrt{\frac{\beta}{\alpha}} \log \left(\frac{\sqrt{ } \beta \cdot \sqrt{ }\left(\alpha \cdot x^{2}+\beta-\gamma^{2}\right)+\gamma \sqrt{ } \alpha \cdot x}{\sqrt{\beta} \cdot \sqrt{ }\left(\alpha x^{2}+\beta-\gamma^{2}\right)-\gamma \sqrt{\alpha \cdot x}}\right)^{2}+\text { Const. }
\end{aligned}
$$

ist, so wird man mittels der obigen Angaben und nach einigen nahe liegenden Umformungen schliefslich zu folgender Gleichung gelangen:

$$
\begin{aligned}
& 2 \sqrt{ }(-a b c) \int_{0}^{c} d x \int_{0}^{\eta} d y \int_{1}^{\zeta} f\left(a x^{2}+b y^{2}+c z^{2}\right) d z \\
& =\frac{1}{2} \pi\left\{\int_{1)}^{a \xi^{2}} f(t) \sqrt[V]{ }(-t) d t+\xi \sqrt{ }(-a) \int_{a \xi^{2}}^{a \xi^{2}+b \eta^{2}+c \zeta^{2}} f(t) d t\right\} \\
& -\int_{a \xi^{2}+b \eta^{2}}^{a \xi^{2}+b \eta^{2}+c \xi^{2}} f(t)\left\{\xi_{V}-a \arccos \frac{\eta \sqrt{ } b}{\sqrt{\left(t-a \xi^{2}\right)}}-\frac{1}{4} \sqrt{ } t \log \left(\frac{\sqrt{ } t \cdot \sqrt{ }\left(t-a \xi^{2}-b \eta^{2}\right)-\xi \eta \sqrt{ }(-a b)}{\sqrt{t} \cdot \sqrt{ }\left(t-a \xi^{2}-b \eta^{2}\right)+\xi \eta \sqrt{ }(-a b)}\right)^{2}\right. \\
& \left.+\frac{1}{4}\left(\eta_{V} / b\right) \log \left(\frac{\sqrt{ }\left(t-a \xi^{2}-b \eta^{2}\right)-\xi \sqrt{ }-a}{\sqrt{ }\left(t-a \xi^{2}-b \eta^{2}\right)+\xi \sqrt{ }-a}\right)^{2}\right\} d t
\end{aligned}
$$

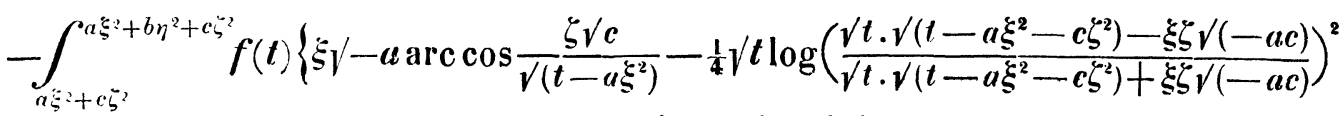

$$
\begin{aligned}
& \left.+\frac{1}{4}(\zeta \sqrt{ } c) \log \left(\frac{\sqrt{ }\left(t-a \xi^{2}-c \zeta^{2}\right)-\xi \sqrt{ }-a}{\sqrt{ }\left(t-a \xi^{2}-c \zeta^{2}\right)+\xi \sqrt{ }-a}\right)^{2}\right\} d t \\
& -\int_{b \eta^{2}+c \zeta^{2}}^{a \xi^{2}+b \eta^{2}+c \zeta^{2}} f(t)\left\{\frac{1}{4}(\eta \sqrt{ } b) \log \left(\frac{\sqrt{ }\left(b \eta^{2}+c \zeta^{2}-t\right)+\zeta \sqrt{ } c}{\sqrt{ }\left(b \eta^{2}+c \zeta^{2}-t\right)-\zeta \sqrt{ } c}\right)^{2}\right. \\
& \left.-\frac{1}{4} \sqrt{ } t \log \left(\frac{\sqrt{ } t \cdot \sqrt{ }\left(b \eta^{2}+c \zeta^{2}-t\right)+\eta \zeta \sqrt{ } b c}{\sqrt{t} \cdot \sqrt{ }\left(b \eta^{2}+c \zeta^{2}-t\right)-\eta \zeta \sqrt{b c}}\right)^{2}+\frac{1}{4}(\zeta \sqrt{ } c) \log \left(\frac{\sqrt{ }\left(b \eta^{2}+c \zeta^{2}-t\right)+\eta \sqrt{ } b}{\sqrt{ }\left(b \eta^{2}+c \zeta^{2}-t\right)-\eta \sqrt{ } b}\right)^{2}\right\} d t .
\end{aligned}
$$

Die Bedingungen, unter welchen diese Formel gilt, sind, gemäfs dem Vorhergehenden, dafs $a$ negativ $a \dot{\xi}^{2}+b \eta^{2}>0, a \dot{\xi}^{2}+c \xi^{2}>0$ sei.

$\mathrm{Zu}$ bemerken ist, dafs sich diese Gleichung aus der in (\$. 5.) für ein positives a gefundenen ableiten läfst, wenn man beachtet, dafs in jener frühern Gleichung alle Bogen, mit Ausnahme des einzigen $\frac{\eta \sqrt{ } b}{\sqrt{ }\left(t-a \xi^{2}\right)}$, imaginür werden, theils weil der Cosinus gröfser als 1, theils weil er selbst imaginär wird; dafs ferner, wo $t=b \eta^{2}$ als Zwischenwerth der Grenzen sich zeigt, der Cosinus unendlich grofs wird und dabei sein Zeichen ändert. Zerlegt man dann die zwei Integrale, bei welchen Dies der Fall ist, mit Rücksicht auf diese Bemerkungen, in zwei andere und verwandelt die imaginären Bogen in reelle Loyarithmen, thut das Gleiche auch bei dem letzten Gliede der Formel (\$. 5.) und dividirt schliefslich die ganze Gleichung mit $\sqrt{ }-1$, so wird man die Formel für ein negalives $a$, übereinstimmend mit der obigen, finden. 
1. Winckler, über die Reduction dreifacher Integrale auf Quadraturen. 15

7.

Wenn in dem Argumente $t$ die Quadrate der Veränderlichen fehlen, also $a, b, c$ Null sind, so werden die eben gefundenen Resultate unbrauchbar und es ist eine directe Bestimmung nöthig.

Es sei also

$$
t=a x y+b x z+c y z .
$$

Die Fläche (Fig. 5.), welcher diese Gleichung angehört, ist, wenn man $a, b, c$ als positiv betrachtet, so lange $\boldsymbol{t}$ positiv bleibt, das eintheilige elliptische $\boldsymbol{H} y$ perboloüd, welches einer Kegelfläche asymptotisch sich nähert, deren Spitze im Anfangspuncte der Coordinaten liegt und von welcher die Coordinaten-Axen drei Erzeugungslinien bilden. Wird $t$ negativ, so giebt die Gleichung das zweitheilige Hyperboloüd, welches, innerhalb jenes Kegels liegend, sich demselben ebenfalls asymptotisch anschliefst. Läfst man also $t$ alle positiven und negativen Werthe durchlaufen, so entsteht ein System von Flächen, welche den ganzen unendlichen Raum ausfüllen; wovon jedoch nur Theile der zweitheiligen Hyperboloïde in den hier zu betrachtenden Raum der positiven Coordinaten fallen. Dieselben zerlegen diesen Raum in vier gesondert zu berechnende Theile, deren bezügliche Grenzen sich wie folgt erstrecken:

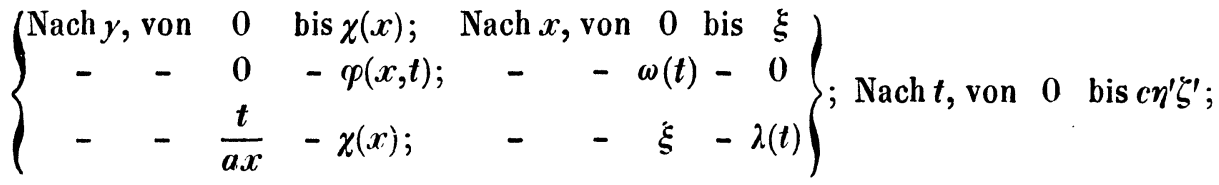

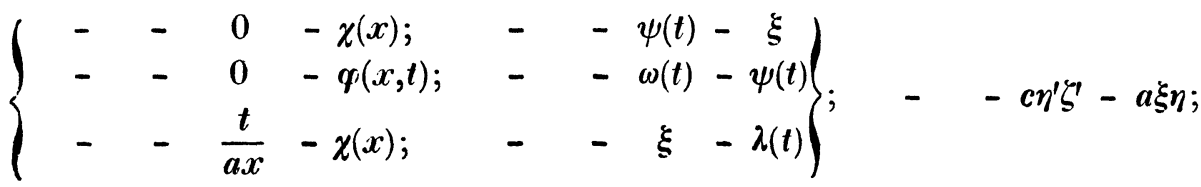

$$
\begin{aligned}
& \left\{\begin{array}{llllll}
- & - & 0 & -\chi(x) ; & - & -\psi(t)-\xi \\
- & - & 0 & -\varphi(x, t) ; & - & -\omega(t)-\psi(t)
\end{array}\right\} ; \quad-\quad-a \xi \eta-b \xi \zeta^{\prime \prime} ; \\
& \text { - } \quad-\varphi(x, t)-\chi(x) ; \quad-\quad-\psi(t)-\xi ; \quad-\quad-b \xi \zeta^{\prime \prime}-a \xi \eta+b \xi \zeta+c \eta \zeta .
\end{aligned}
$$

Ferner ist:

$$
d S=\frac{d t}{c} \int(\log (b x+c y)+\text { Const. }) d x .
$$

Die weitere Ausführung möge z. B. auf den Fall bezogen werden, in welchem die Grenzen des dreifachen Integrals constant sind. Dann ist:

$$
\begin{aligned}
\varphi(x, t) & =\frac{t-b \zeta x}{a x+c \zeta}, & \omega(t) & =\frac{t}{b \zeta}, \\
\lambda(t) & =\frac{t}{a \eta}, & \psi(t) & =\frac{t-c \eta \zeta}{a \eta+b \zeta}
\end{aligned}
$$


16 1. Winckler, über die Reduction dreifacher Integrale auf Quadraturen.

ferner, wenn $\alpha$ und $\beta$ posiliv sind:

$$
\int \log \left(\alpha x^{2}+\beta\right) d x=x \log \left(\alpha x^{2}+\beta\right)+2 \sqrt{\frac{\beta}{\alpha}} \operatorname{arctg}\left(x / \frac{\alpha}{\beta}\right)-2 x+\text { Const. }
$$

Mittels dieser Angaben erhält man, nach einigen Reductionen, die symmetrische Gleichung

$$
\int_{0}^{\xi} d x \int_{0}^{\eta} d y \int_{0}^{\zeta} f(a x y+b x z+c y z) d z
$$

$=\int_{0}^{a \xi \eta} f(t)\left\{\frac{\xi}{c} \log \frac{a b \xi^{2}+c t}{a \xi(b \xi+c \eta)}+\frac{\eta}{b} \log \frac{a c \eta^{2}+b t}{a \eta(c \eta+b \xi)}+2 \sqrt{\frac{t}{a b c}} \operatorname{arctg}\left(\sqrt{\frac{b c}{a t}} \cdot \frac{a \xi \eta-t}{b \xi+c \eta}\right)\right\} d t$

$+\int_{1}^{b \xi \zeta} f(t)\left\{\frac{\zeta}{a} \log \frac{b c^{2}+a t}{b \zeta(c \zeta+a \xi)}+\frac{\xi}{c} \log \frac{a b \xi^{2}+c t}{b \xi(a \xi+c \zeta)}+2 \sqrt{\frac{t}{a b c}} \operatorname{arctg}\left(\sqrt{\frac{a c}{b t}} \cdot \frac{b \xi \zeta-t}{c \zeta+a \xi}\right)\right\} d t$

$+\int_{0}^{c \eta \zeta} f(t)\left\{\frac{\eta}{b} \log \frac{a c \eta^{2}+b t}{c \eta(a \eta+b \zeta)}+\frac{\zeta}{a} \log \frac{b c \zeta^{2}+a t}{c \zeta(b \zeta+a \eta)}+2 \sqrt{\frac{t}{a b c}} \operatorname{arctg}\left(\sqrt{\frac{a b}{c t}} \cdot \frac{a \eta \zeta-t}{a \eta+b \zeta}\right)\right\} d t$

$+\int_{1}^{a \xi \eta+b \xi \zeta+c \eta \xi} f(t)\left\{\frac{\xi}{c} \log \frac{(a \xi+c \zeta)(b \xi+c \eta)}{a b \xi^{2}+c t}+\frac{\eta}{b} \log \frac{(c \eta+b \xi)(a \eta+b \zeta)}{a c \eta^{2}+b t}+\frac{\zeta}{a} \log \frac{(b \zeta+a \xi)(c \zeta+a \eta)}{b c \zeta^{2}+a t}\right.$

$\left.-2 \sqrt{\frac{t}{a b c}}\left(\operatorname{arctg} \xi \sqrt{\frac{a b}{c t}}+\operatorname{arctg} \eta / \frac{a c}{b t}+\operatorname{arctg} \zeta \sqrt{\frac{b c}{a t}}-\frac{1}{2} \pi\right)\right\} d t$.

Sind die Grenzen $\xi, \eta$, $\zeta$ insgesammt unendlich, so heben sich die logarithmischen Ausdrücke gegenseitig auf und es bleiben nur die Bogengrö/sen übrig, von welchen jede einzelne sich auf $\frac{1}{2} \pi$ reducirt. Man findet also, wenn $f(t)$ für $t=\infty$ verschwindet:

$$
\int_{0}^{\infty} d x \int_{0}^{\infty} d y \int_{0}^{\infty} f(a x y+b x z+c y z) d z=\frac{\pi}{\sqrt{a b c}} \int_{0}^{\infty} f(t) \cdot \sqrt{ } t \cdot d t .
$$

Vergleicht man diese Formel mit der am Schlusse von (\$.5.) angeführten, so zeigt sich noch, dafs unter den jetzigen Voraussetzungen

$$
\int_{11}^{\infty} d x \int_{1}^{\infty} d y \int_{0}^{\infty} f(a x y+b x z+c y z) d z=4 \int_{0}^{\infty} d x \int_{0}^{\infty} d y \int_{0}^{\infty} f\left(a x^{2}+b y^{2}+c z\right) \cdot d z
$$

ist. Wie zu verfahren sei, wenn einer der Coëfficienten $a, b$, c negativ ist, bedarf keiner besondern Auseinandersetzung.

8.

Wenn das Argument $t$ der Quotient zweier Functionen zweiten Grades von $x, y, z$ ist, so entspricht der Gesammtheit aller Werthe von $t$ ein System von Flächen zweiter Ordnung, welche sich, im Allgemeinen, in einer räumlichen Curve vierter Ordnung schneiden. Die Gleichungen dieser Curve er- 
geben sich, wenn man Zähler und Nenner von $t$, jeden für sich, gleich Null setzt. Wie leicht zu erachten, ist diese Curve sehr oft imaginär oder liegt im Unendlichen, oder hat auch nur eine einzige reelle Projection. Die Untersuchung der solchen Argumenten entsprechenden Integrale ist sehr weitläuftig und die Resultate werden überaus complicirt. Als Beispiele wollen wir die folgenden besondern Fälle näher betrachten.

Es sei

$$
t=\frac{a x^{2}+b y^{2}+c z^{2}+m}{\alpha x^{2}+\beta y^{2}+\gamma s^{2}+\mu}
$$

und darin seien alle Coëfficienten positiv und aufserdem sei

$$
\frac{a}{\alpha}>\frac{b}{\beta}>\frac{c}{\gamma}>\frac{m}{\mu} \text {. }
$$

Dann stellt die Gleichung:

$$
(\alpha t-a) x^{2}+(\beta t-b) y^{2}+(\gamma t-c) z^{2}+\mu t-m=0,
$$

wenn man $t$ (Fig. 6.) von dem Werthe $\frac{m}{\mu}$ an wachsend annimmt, eine Reihe von Flächen dar, welche für $t=\frac{m}{\mu}$ mit dem Puncle anfängt, in das dreiaxige Ellipsoüd übergeht, für $t=\frac{c}{\gamma}$ einen zur $z$-Axe parallelen elliptischen Cylinder und dann das eintheilige Hyperboloäd giebt, welches sich für $t=\frac{b}{\beta}$ auf einen zur $y$-Axe parallelen hyperbolischen Cylinder reducirt. Von hier an geht die Fläche in das zweitheilige Hyperbolö̈d über, welches für $t=\frac{a}{\alpha}$ im Unendlichen mit der $x$-Axe zusammenfältt. Während also $t$ alle Werthe von $\frac{m}{\mu}$ bis $\frac{a}{\alpha}$ durchläuft (und andere können nicht vorkommen), entstehen Flächen, welche den ganzen unendlichen Raum ausfüllen. Die Durchschnittslinie aller dieser Flächen ist, unter den zu Grunde gelegten Annahmen, imaginär.

In dem besondern Falle, wenn $m$ und $\mu$ Null sind, gehen die Flächen, von welchen oben die Rede war, in Kègelfü̈chen über, deren Spitze im Anfangspuncte liegt und deren Axe die $x$-Axe ist. Und zwar reduciren sich dieselben, unter den obigen Voraussetzungen, für $t=\frac{a}{\alpha}$ auf die $x$-Axe, sind für die folgenden, kleineren Werthe von $t$ elliptische Kegel und für $t=\frac{b}{\beta}$ zwei sich in der $y$-Axe schneidende Ebenen. Für noch kleinere Werthe Crelle's Journal f. d. M. Bd. L. Heft 1. 
18 1. Winckler, über die Reduction dreifacher Integrale auf Quadraturen.

von $t$ entstehen hyperbolische Kegel, welche sich schliefslich für $t=\frac{c}{\gamma}$ auf die $z$-Axe reduciren.

Dies vorausgesetzt, sind die Grenzwerthe der sechs einzelnen Theile, in welche das entsprechende dreifache Integral zerfällt, wie folgt zu nehmen:

Nach $y$, von 0 bis $\varphi(x, t) ; \quad$ Nach $x$, von $0 \quad$ bis $\omega(t)$;

Nach $t$, von $\frac{c}{\gamma}$ bis $\frac{b \eta^{\prime 2}+c \zeta^{\prime 2}}{\beta \eta^{\prime 2}+\gamma \zeta^{\prime 2}}$

\{Nach $y$, von 0 bis $\chi(x)$;

Nach $x$, von 0 bis $\psi(t)$

$\{\quad-0-\varphi(x, t) ; \quad-\quad-\psi(t)-\omega(t)\}$

Nach $t$, von $\frac{b \eta^{\prime 2}+c \zeta^{\prime 2}}{\beta \eta^{\prime 2}+\gamma \zeta^{\prime 2}}$ bis $\frac{a \xi^{2}+b \eta^{2}+r \zeta^{2}}{\alpha \xi^{3}+\beta \eta^{2}+\gamma \zeta^{2}}$

\{Nach $y$, von 0 bis $\chi(x)$;

Nach $x$, von 0 bis $\xi\}$

Nach $t$, von $\frac{a \xi^{2}+b \eta^{2}+c \xi^{2}}{\alpha \xi^{2}+\beta \eta^{2}+\gamma \zeta^{2}}$ bis $\frac{a \xi^{2}+c \xi^{\prime \prime 2}}{\alpha \xi^{2}+\gamma \xi^{\prime \prime 2}}$

Nach $y$, von 0 bis $\chi(x)$; $\quad$ Nach $x$, von 0 bis $\xi$;

Nach $t$, von $\frac{a \xi^{2}+c \zeta^{\prime \prime 2}}{\alpha \xi^{2}+\gamma \xi^{1{ }^{2}}}$ bis $\frac{b}{\beta}$;

$\left\{\right.$ Nach $y$, von 0 bis $x /-\frac{\alpha t-a}{\beta t-b} ; \quad$ Nach $x$, von 0 bis $\left.\lambda(t)\right\}$;

- 0 - $\chi(x)$;

$-\lambda(t)-\xi)$

Nach $t, \operatorname{von} \frac{b}{\beta}$ bis $\frac{a \xi^{2}+b \eta^{2}}{\alpha \zeta^{2}+\beta \eta^{2}}$

Nach $y$, von 0 bis $x /-\frac{\alpha t-a}{\beta t-b}$; Nach $x$, von 0 bis $\xi$;

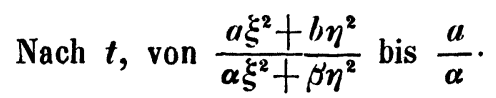

Ferner ist:

$$
\left(\frac{d z}{d t}\right)=\frac{(\alpha c-a \gamma) x^{2}+(\beta c-b \gamma) y^{2}}{2(\gamma t-c)^{\frac{3}{2}} \cdot \sqrt{ }\left((a-\alpha t) x^{2}+(b-\beta t) y^{2}\right)} .
$$

Um daraus $d \boldsymbol{S}$ in reeller Form zu finden, mufs man bei der Integration nach $y$ unterscheiden, ob $t<$ oder $>\frac{b}{\beta}$. ist. Im ersten Falle findet man logurithmische, im letztern Bogen-Ausdrücke.

9.

Für die eben betrachtete Gleichung:

$$
(\alpha t-a) x^{2}+(\beta t-b) y^{2}+(\gamma t-c) z^{2}+\mu t-m=0
$$

wollen wir die Voraussetzungen dahin abändern, dafs nun $m$ und, negativ 
und aufserdem

$$
\frac{a}{a}>\frac{m}{\mu}>\frac{b}{\beta}>\frac{c}{\gamma}
$$

sein soll. Alsdann existirt in der That eine gemeinschaftliche Durchschnillslinie aller Flächen. Die Gleichungen derselben sind:

$$
\begin{aligned}
& a x^{2}+b y^{2}+c z^{2}+m=0, \\
& \alpha x^{2}+\beta y^{2}+\gamma z^{2}+\mu=0 .
\end{aligned}
$$

Wenn man hieraus die Gleichungen zwischen je zwei der Veränderlichen ableitet, so findet sich, dafs die Projection jener räumlichen Curve, auf $x y$ Theile eine Ellipse, auf $x z$ Theile eine Hyperbel und auf zy, eine vollständige Ellipse ist. Diese drei Curven sind zugleich die Leitlinien von Cylinderflächen, deren Axen beziehungsweise die Axen der $z, y, x$ sind.

Wegen des Verlaufes aller, den aufeinander folgenden Werthen von $t$ entsprechenden Flächen genügt die Bemerkung, dafs man für $-\infty<t<\frac{c}{\gamma}$ und für $\frac{a}{\alpha}<t<+\infty$ dreiaxige Ellipsoüde; für $t=\frac{c}{\gamma}$ den bemerkten zur $z$-Axe parallelen Cylinder ; für $\frac{c}{\gamma}<t<\frac{b}{\beta}$ eintheilige Hyperboloüden (deren imaginäre Halb-Axen in der $z$-Axe liegen) und für $t=\frac{b}{\beta}$ den schon erwähnten, zur $y$-Axe parallelen Cylinder erhält; dafs ferner für $\frac{b}{\beta}<t<\frac{m}{\mu}$ zweitheilige nach der $x$-Axe sich öffnende Hyperboloüden; für $t=\frac{m}{\mu}$ ein elliptischer Kegel (dessen Spitze im Anfangspunct liegt); für $\frac{m}{\mu}<t<\frac{a}{\alpha}$ wieder eintheilige Hyperbolö̈den (deren imaginäre Halb-Axen in der $x$-Axe liegen) entstehen; und endlich dafs für $t=\frac{u}{\alpha}$ der bereits genannte, zur $x$-Axe parallele Cylinder zum Vorschein kommt.

10.

Ist

$$
t=\frac{a x y+b x z+c y z}{\alpha x y+\beta x z+\gamma y z}
$$

so sind die allen möglichen Werthen von $t$ entsprechenden Flächen insgesammt Kegelfächen, welche sich in den durch die beiden Gleichungen:

$$
\begin{aligned}
& a x y+b x z+c y z=0, \\
& \alpha x y+\beta x z+\gamma y z=0
\end{aligned}
$$

gegebenen Linien schneiden. 
20 1. Winckler, über die Reduction dreifacher Integrale auf Quadraturen.

Es ist leicht zu sehen und nicht näher auszuführen nöthig, daf́s diese Gleichungen vier gerade Linien (Fig. 7.) darstellen, von welchen drei die Coordinaten-Axen selbst, während die Projections-Gleichungen der vierten

$$
\begin{gathered}
(a \beta-\alpha b) x+(a \gamma-\alpha c) y=0 ; \quad(b \gamma-\beta c) z+(b \alpha-\beta a) x=0 ; \\
(c \alpha-\gamma a) y+(c \beta-\gamma b) \approx=0
\end{gathered}
$$

sind. Ferner sieht man aus der Gleichung der Flächen:

$$
(\alpha t-a) x y+(\beta t-b) x z+(\gamma t-c) y z=0,
$$

dafs sich dieselben für $t=\frac{a}{a}, \frac{b}{\beta}, \frac{c}{\gamma}$ jedesmal auf swei Ebenen reduciren, wovon eine durch jene vierte Gerade und beziehungsweise durch die $z, y, x$-Axe geht und die andern mit der $x y, x z, y z-$ Ebene zusammenfällt. Die krummen Flächen reduciren sich also sechsmal auf Ebenen und stellen die Gesammtheit aller Kegelflïchen zweiter Ordnung dar, welche durch vier, im Anfangspuncte sich schneidende gerade Linien gehen. Die ausführliche Aufstellung der Resultate findet jedoch hier nicht Raum.

Aufser den bisher für $t$ angenommenen Ausdrücken lassen sich noch beliebig viele andere irrationale und transcendente bezeichnen, für welche sich die Reduclion des Integrals allgemein darstellen läfst und auf welche das bisherige Verfahren anwendbar bleibt. Hier möge nur des Falles Erwähnung geschehen, in welchem

$$
t=\boldsymbol{a} e^{\alpha x}+b e^{\beta y}+c e^{\gamma z}
$$

ist. Die dieser Gleichung angehörige transcendente Fläche kehrt, wenn alle Coëfficienten positiv sind, ihre geschlossene Seite dem Raume der positiven Halb-Axe zu und geht für $t=a+b+c$ durch den Anfangspunct. Für kleinere Werthe von $t$ liegt kein Punct der Fläche mehr in jenem Raume.

Die Aufstellung des Systems der Grenzwerthe und der Reductionsformel hat keine Schwierigkeit. Nimmt man z. B. an, die obern Grenzen des dreifachen Integrals seien insgesammt unendlich, so erhält man nur ein einziges Integral, welches wie folgt zu nehmen ist:

Nach $y$, von 0 bis $\frac{1}{\beta} \log \frac{t-a e^{\alpha x}-c}{b} ; \quad$ Nach $x$, von 0 bis $\frac{1}{a} \log \frac{t-b-c}{a}$;

Da ferner

Nach $t$, von $a+b+c$ bis $\infty$.

$$
\left(\frac{d z}{d 1}\right)=\frac{1}{\gamma} \cdot \frac{1}{t-u e^{\alpha . x}-6 e^{\beta y}}
$$


1. Winckler, ̈̈ber die Reduction dreifacher Integrale auf Quadruturen. 21 ist, so geht das dreifache Integral in

$$
\frac{1}{\beta \gamma} \int_{a+b+c}^{\infty} f(t) d t \int_{0}^{\frac{1}{\alpha} \log \frac{t-b-c}{a}} \frac{d x}{t-a e^{\alpha x}} \log \frac{\left(t-a e^{\alpha x}-b\right)\left(t-a e^{\alpha x}-c\right)}{b c}
$$

über, oder man erhält nach einiger Transformation, und wenn man dann auch die Integrationsfolge ändert:

$$
\begin{gathered}
\int_{0}^{\infty} d x \int_{1}^{\infty} d y \int_{0}^{\infty} f\left(a e^{\alpha x}+b e^{\beta y}+c e^{\gamma z}\right) d z \\
=\frac{1}{\alpha \beta \gamma} \int_{a+b+c}^{\infty} f(t) d t \int_{a+b+c}^{t} \frac{d z}{(z-a)(t+a-z)} \log \frac{(z-a-b)(z-a-c)}{b c} \\
=\frac{1}{\alpha \beta \gamma} \int_{0}^{\infty} \frac{d z}{a+c+z} \log \frac{(a+z)(c+z)}{a c} \int_{0}^{\infty} f(a+b+c+t+z) \frac{d t}{b+t} .
\end{gathered}
$$

Dafs die Integrale rechts, wie nach $\alpha, \beta, \gamma$, so auch nach $a, b, c$ symmetrische Functionen sein müssen, versteht sich, geht übrigens auch aus den Gleichungen (A. $\$$. 1.) hervor, wenn man sie auf das durch die oben angegebenen Grenzen (nach $y$ und $x$ ) bestimmte Doppel-Integral anwendet. Diese Symmetrie läfst sich übrigens auf ähnliche Weise darstellen, wie $九$. B. diejenige des einfachen Integrals

$$
\int_{a+b}^{t} \frac{d z}{(z-a)(t+a-z)}
$$

ohne dafs die Ausführung der Integration nöthig wäre.

11.

Aus den oben angeführten Reductionsformeln unseres dreifachen Integrals mögen noch die folgenden speciellen Resultate abgeleitet werden.

Es sei $a=b=c, \alpha=\beta=\gamma$ und $f(t)=e^{-t}$, so ergiebt sich, mit Rücksicht auf die bekannten Formeln,

$$
\operatorname{li}\left(e^{-c k}\right)=\int_{0}^{e^{-c k}} \frac{d x}{\log x}=-\int_{1}^{\infty} e^{-c k x} \frac{d x}{x}=-e^{-c k} \int_{0}^{\infty x} e^{-c k} \frac{d x}{x+c},
$$

die sehr bemerkenswerthe Gleichung

$$
\int_{0}^{\infty} e^{-z} \log \frac{(z+a)(z+b)}{a b} \cdot \frac{d z}{z+a+b}=e^{a+b} \cdot \operatorname{li}\left(e^{-a}\right) \operatorname{li}\left(e^{-b}\right),
$$

welche sich jedoch auch durch ein anderes Verfahren erlangen läfst. Setzt man hierin $a k, b k, k z$ statt $a, b, z$ und reducirt, so erhält man weiter:

$$
\int_{0}^{\infty} \frac{e^{-k z} d z}{z+a+b} \log (z+a)(z+b)=e^{(a+b) k}\left\{\operatorname{li}\left(e^{-a k} \operatorname{li}\left(e^{-b k}\right)-\log a b \cdot \operatorname{li}\left(e^{-(a+b) k}\right)\right\} .\right.
$$


Durch theilweises Integriren und mit Berücksichtigung der Gleichung

$$
\int_{1}^{\infty} e^{-k x} \log (x+c) d x=\frac{1}{k}\left(\log c-e^{c k} \operatorname{li}\left(e^{-c k}\right)\right)
$$

ergiebt sich hieraus noch folgendes Resultat:

$$
\int_{0}^{\infty} e^{-k z}\left(\frac{1}{z+a}+\frac{1}{z+b}\right) \log (z+a+b) d z
$$

$=(1+\log a \log b) \log (a+b)-e^{-(a+b) k}\left\{\operatorname{li}\left(e^{-a k}\right) \operatorname{li}\left(e^{-b k}\right)+(1-\log a b) \operatorname{li}\left(e^{-(a+b) h}\right)\right\}$. I. s. f.

12.

Fügt man dem $f(t)$ noch eine vollständig gegebene Function von $x, y, \approx$ als Factor bei, so erleiden die Grenzen, welche, wie man weifs, aus der Beschaffenheit des Arguments $t$ ihre Bestimmung erhalten, keinerlei Änderung, während jedoch die betreffenden Reductionsformeln eine beträchtliche Verallgemeinerung erfahren. Da das Verfahren dasselbe bleibt, wie bisher, so möge nur der Fall berührt werden, welcher sich auf die lineare Form des Arguments ( $\$$. 2.) bezieht und wo der Einfachheit wegen angenommen werden mag, die Grenzen des dreifachen Integrals seien insgesammt constant. Die entsprechende Gleichung kann entweder durch Umgestaltung der in (\$. 2.) gegebenen Grenzen, oder, was noch bequemer ist, mit Rücksicht auf die in (\$.1.) angeführte Transformations-Gleichung (A.), unmittelbar aus der (Fig. 8.) abgelesen werden, wo der Raum so abgetheilt ist, dafs das Resultat sogleich in seiner symmetrischen Form sich zeigt.

Bei den vorliegenden Betrachtungen besteht überhaupt ein wesentlicher Nutzen der geometrischen Repräsentation darin, dafs sich sogleich aus der Figur abnehmen läfst, welches die einfachste und eleganteste Darstellung des entsprechenden Integrals sei. Insbesondere entspricht einer symmetrischen Figur immer auch ein symmetrischer analytischer Ausdruck. Setzt man zur Abkürzung:

$$
\begin{gathered}
F\left(x, y, \frac{t-a x-b y}{c}\right)=\mathrm{F}(x, y) ; \quad \boldsymbol{F}\left(x, \frac{t-a x-c z}{b}, z\right)=\mathrm{F}(x, z) ; \\
F\left(\frac{t-b y-c z}{a}, y, z\right)=\mathrm{F}(\dot{y}, z),
\end{gathered}
$$

so ergiebt sich

$$
\int_{0}^{\xi} d x \int_{0}^{\eta} d y \int_{0}^{\zeta} f(a x+b y+c z) \cdot F(x, y, z) d z
$$


1. Winckler, über dic Reduction dreifacher Integrale auf Quadraturen. 23

$$
\begin{aligned}
& =\frac{1}{c} \int_{1}^{a \xi} f(t) d t \int_{1}^{\frac{t-a \xi}{b}} d y \int_{\xi}^{\frac{t-b y}{a}} \mathrm{~F}(x, y) d x+\frac{1}{a} \int_{11}^{b \eta} f(t) d t \int_{0}^{\frac{t-b \eta}{c}} d z \int_{\eta}^{\frac{t-c z}{b}} \mathrm{~F}(y, z) d y \\
& +\frac{1}{b} \int_{1}^{c \zeta} f(t) d t \int_{11}^{\frac{t-c \zeta}{a}} d x \int_{\zeta}^{\frac{t-a x}{c}} \mathrm{~F}(x, z) d z \\
& -\frac{1}{c} \int_{1}^{a \xi+b \eta} f(t) d t \int_{\eta}^{\frac{t-a \xi}{b}} d y \int_{\xi}^{\frac{t-b y}{a}} \mathrm{~F}(x, y) d x \\
& -\frac{1}{a} \int_{0}^{b \eta+c \zeta} f(t) d t \int_{\zeta}^{\frac{t-b \eta}{c}} d z \int_{\eta}^{\frac{1-c z}{b}} \mathrm{~F}(y, z) d y \\
& -\frac{1}{b} \int_{\Theta}^{a \xi+c \zeta} f(t) d t \int_{\xi}^{\frac{t-c \zeta}{a}} d x \int_{\zeta}^{\frac{t-a x}{c}} \mathrm{~F}(x, z) d z \\
& +\frac{1}{c} \int_{0}^{a \dot{\xi}+b \eta+c \zeta} f(t) d t \int_{\eta}^{\frac{t-a \xi-c \zeta}{b}} d y \int_{\zeta}^{\frac{t-b y-c \zeta}{a}} \mathrm{~F}(x, y) d x .
\end{aligned}
$$

Das letzte Glied hat hier keine symmetrische Form, aber es wäre leicht, demselben mittels der Gleichung ( $\boldsymbol{A}$. des $\$$. 1.) eine solche Form zu geben; wobei es jedoch durch $d r e i$ dem Werthe nach gleiche Integrale ausgedrückt werden würde.

13.

Als Beispiel für diese Formel wollen wir

$$
\boldsymbol{H}(\boldsymbol{x}, \boldsymbol{y}, \boldsymbol{z})=\boldsymbol{e}^{\alpha x+\beta y+\gamma z}
$$

setzen. Führt man die Integrationen aus und setzt zur Abkürzung:

$$
\beta c-b \gamma=a_{1}, \quad \gamma a-c \alpha=b_{1}, \quad \alpha b-a \beta=c_{1},
$$

so wird sich folgende Formel finden:

$$
\begin{gathered}
\int_{0}^{\xi \xi} d x \int_{0}^{\eta \eta} d y \int_{1}^{\zeta} f(a x+b y+c z) \cdot e^{\alpha x+\beta y+\gamma z} d z= \\
-\frac{1}{a_{1} b_{1} c_{1}}\left\{a a_{1} \int_{0}^{a \xi} f(t) e^{\frac{\alpha}{a} t} d t+b b_{1} \int_{0}^{b \eta} f(t) e^{\frac{\beta}{b} t} d t+c c_{1} \int_{0}^{c \zeta} f(t) e^{\frac{\gamma}{c} t} d t\right\} \\
+\frac{a}{b_{1} c_{1}}\left\{\int_{b \eta}^{a \xi+b \eta} f(t) e^{\frac{a t-c_{1} \eta}{a}} d t-\int_{b \eta+c \zeta}^{\alpha \xi+b \eta+c \zeta} f(t) e^{\frac{\alpha t-c_{1} \eta+b_{1} \zeta}{a}} d t+\int_{c \zeta}^{a \xi+c \zeta} f(t) e^{\frac{\alpha t+b_{1} \zeta}{a}} d t\right\} \\
+\frac{b}{c_{1} a_{1}}\left\{\int_{c \zeta}^{b \eta+c \zeta} f(t) e^{\frac{\beta t-a_{1} \zeta}{b}} d t-\int_{a \xi+c \zeta}^{a \xi+b \eta+c \zeta} f(t) e^{\frac{\beta t-a_{1} \zeta+c_{1} \xi}{b}} d t+\int_{a \xi}^{a \xi+b \eta} f(t) e^{\frac{\beta t+c_{1} \xi}{b}} d t\right\} \\
+\frac{c}{a_{1} b_{1}}\left\{\int_{a \xi}^{a \xi+c \zeta} f(t) e^{\frac{\gamma t-b_{1} \xi}{c}} d t-\int_{a \xi+b \eta}^{a \xi+b \eta+c \zeta} f(t) e^{\frac{\gamma t-b_{1} \xi+a_{1} \eta}{c}} d t+\int_{b \eta}^{b \eta+c \zeta} f(t) e^{\frac{\gamma t+a_{1} \eta}{c}} d t\right\} .
\end{gathered}
$$


24 1. Winckler, über die Reduction dreifacher Integrale anf Quadruturen.

Es sei hierin $\xi=\eta=\zeta=\infty$, so folgt:

$$
\begin{gathered}
\int_{0}^{\infty} d x \int_{0}^{\infty} d y \int_{0}^{\infty} f(a x+b y+c s) e^{\alpha x+\beta y+\gamma z} d z \\
=\frac{a}{(\alpha c)-a \beta)(\alpha c-a \gamma)} \int_{1}^{\infty} f(t) e^{\frac{\alpha}{\alpha} t} d t+\frac{b}{(\beta a-b \alpha)(\beta c-b \gamma)} \int_{11}^{\infty} f(t) e^{\frac{\beta}{b} t} d t \\
\quad+\frac{c}{(\gamma a-c \alpha)(\gamma b-c \beta)} \int_{0}^{\infty} f(t) e^{\frac{\gamma}{c} t} d t .
\end{gathered}
$$

Ändert man die Zeichen von $\alpha, \beta, \gamma$ und sieht dann die Coëfficienten als positiv an; setzt ferner

$$
f(t)=\frac{1}{t+m}
$$

und bemerkt, dafs sich dann die drei Bestandtheile der Formel durch IntegralLogarithmen ausdrücken lassen, so ergiebt sich:

$$
\begin{gathered}
\int_{1}^{\infty} d x \int_{0}^{\infty} d y \int_{0}^{\infty} \frac{e^{-(\alpha x+\beta y+\gamma z)}}{a x+b y+c z+m} d z \\
=-\left\{\frac{a e^{\frac{\alpha}{a} m} \operatorname{li}\left(e^{-\frac{\alpha}{a} m}\right)}{(\alpha b-a \beta)(\alpha c-a \gamma)}+\frac{b e^{\frac{\beta}{b} m} \operatorname{li}\left(e^{-\frac{\beta}{b} m}\right)}{(\beta a-b \alpha)(\beta c-b \gamma)}+\frac{c e^{\frac{\gamma}{c} m} \operatorname{li}\left(e^{-\frac{\gamma}{c} m}\right)}{(\gamma a-c \alpha)(\gamma b-c \beta)}\right\} .
\end{gathered}
$$

Für ein zweites Beispiel sei:

$$
f(t)=t^{n}
$$

Erwägt man, dafs, wenn wieder $\alpha, \beta, \gamma$ negativ angenommen werden, die drei Integrale der allgemeinen Formel sich auf

$$
\int_{0}^{\infty} t^{n} e^{-t} d t=\Gamma(n+1)
$$

reduciren lassen, so wird sich alsbald die folgende Formel finden:

$$
\begin{gathered}
\int_{0}^{\infty} d x \int_{0}^{\infty} d y \int_{1}^{\infty}(a x+b y+c z)^{n} e^{-(\alpha x+\beta y+\gamma z)} d z \\
I_{(n+1)}\left\{\frac{\alpha}{(\alpha b-a \beta)(\alpha c-a \gamma)}\left(\frac{a}{\alpha}\right)^{n+2}+\frac{\beta}{(\beta a-b \alpha)(\beta c-b y)}\left(\frac{b}{\beta}\right)^{n+2}+\frac{\gamma}{(\gamma a-c \alpha)(\gamma b-c \beta)}\left(\frac{c}{\gamma}\right)^{n+2}\right\} .
\end{gathered}
$$

Endlich setze man noch:

$$
f(t)=\log (t+m)
$$

und nehme wieder an, es seien $\alpha, \beta, \gamma$ negativ. Dann findet sich die 
Gleichung:

$$
\begin{aligned}
& \int_{i}^{\infty} d x \int_{i}^{\infty} d y \int_{i}^{\infty} e^{-(\alpha x+\beta \gamma+\gamma z)} \log (a x+b y+c z+m) d z=\frac{\log m}{\alpha \beta \gamma} \\
& -\left\{\frac{\alpha e^{\frac{\alpha}{a} m} \operatorname{li}\left(e^{-\frac{\alpha}{a} m}\right)}{(\alpha b-a \beta)(\alpha c-a \gamma)}\left(\frac{a}{\alpha}\right)^{2}+\frac{\beta e^{\frac{\beta}{b} m} \operatorname{li}\left(e^{-\frac{\beta}{b} m}\right)}{(\beta a-b \alpha)\left(\rho^{\prime} c-b \gamma\right)}\left(\frac{b}{\beta}\right)^{2}+\frac{\gamma e^{\frac{\gamma}{c} m} \operatorname{li}\left(e^{-\frac{\gamma}{c} m}\right)}{(\gamma b-c \beta)(\gamma a-c \alpha)}\left(\frac{c}{\gamma}\right)^{2}\right\} . \\
& \text { U. s. w. }
\end{aligned}
$$

14.

In der allgemeinen Formel (\$.12.) werde ferner

$$
F^{\prime}(x, y, z)=\frac{1}{(\alpha x+\mu y+\gamma z+\mu)^{n}}
$$

gesetzt. Dann ist, wenn $a_{1}, b_{1}, c_{1}$ dieselbe Bedeutung wie oben behalten:

$$
\begin{aligned}
& (n-1)(n-2) \int_{0}^{\xi} d x \int_{0}^{n} d y \int_{i}^{5} \frac{f(a x+b y+c z)}{(\alpha x+\beta y+\gamma z+\mu)^{n}} d z \\
& =-\left\{\frac{a^{n-1}}{b_{1} c_{1}} \int_{0}^{a \xi} \frac{f(t) d t}{(\alpha t+a \mu)^{n-2}}+\frac{b^{n-1}}{c_{1} a_{1}} \int_{0}^{\cdot b \eta} \frac{f(t) d t}{(\beta t+b \mu)^{n-2}}+\frac{c^{n-1}}{a_{1} b_{1}} \int_{0}^{c c} \frac{f(t) d t}{(\gamma t+c \mu)^{n-2}}\right\}
\end{aligned}
$$

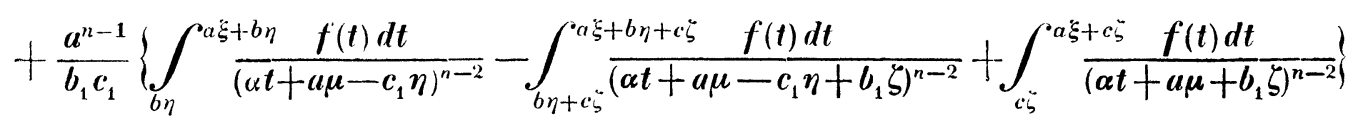

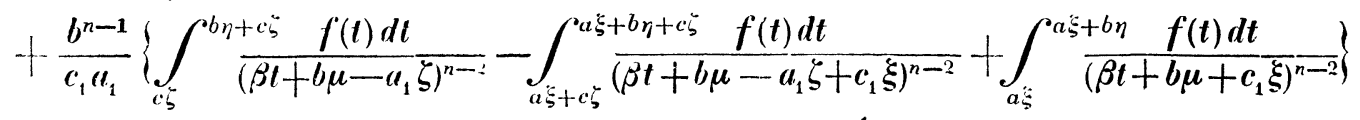

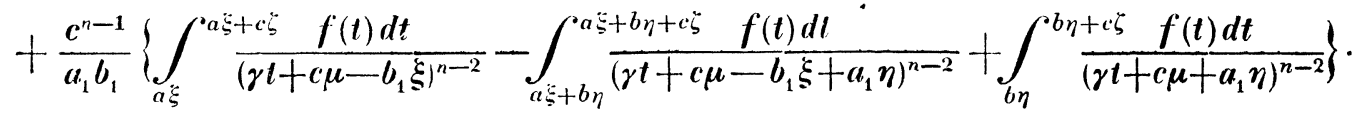

Ist hier $\xi=\eta=\zeta=\infty$, so erhält man:

$$
\begin{gathered}
(n-1)(n-2) \int_{1}^{\infty} d x \int_{0}^{\infty} d y \int_{0}^{\infty} \frac{f(a x+b y+c z)}{(\alpha x+\beta y+\gamma z+\mu)^{n}} d z \\
=\frac{a^{n-1}}{(\alpha b-a \beta)(\alpha c-a \gamma)} \int_{1}^{\infty} \frac{f(t) d t}{(\alpha t+a \mu)^{n-2}}+\frac{b^{n-1}}{(\beta a-b \alpha)(\beta c-b \gamma)} \int_{0}^{\infty} f(t) d t \\
\quad+\frac{c^{n-1}}{(\beta t+b \mu)^{n-2}} \\
\int_{0}^{\infty} \frac{f(t) d t}{(\gamma t+c \mu)^{n-2}} .
\end{gathered}
$$

Für die Werthe 1 und 2 von $n$ werden diese Formeln unbrauchbar und die Reduction mufs auf directe Weise geschehen.

Setzt man in der letztern Gleichung

$$
f(t)=t^{m}
$$

Crelle's Journal f. d. M. Bd. L. Heft 1. 
1. Winclkler, ̈̈ber die Reduction dreifacher Integrale auf Quadraturen.

und beachtet, dafs

$$
\int_{0}^{\infty} \frac{t^{m} d t}{(p t+q)^{n-2}}=\frac{1}{p^{m+1} q^{n-m-3}} \cdot \frac{\Gamma(m+1) \Gamma(n-m-3)}{\Gamma(n-2)}
$$

ist, so zeigt sich vor Allem, dafs das dreifache Integral einen endlichen Werth hat, wenn

$$
n>2 \text { und } \quad m<n-3
$$

ist, und man findet unter dieser Voraussetzung folgendes Resultat:

$$
\begin{gathered}
\int_{0}^{\infty} d x \int_{0}^{\infty} d y \int_{1}^{\infty} \frac{(a x+b y+c z)^{m}}{(\alpha x+\beta y+\gamma z+\mu)^{n}} d z \\
=\frac{1}{\mu^{n-m-3}} \frac{\Gamma(m+1) \Gamma(n-m-3)}{(n-1)(n-2) \Gamma(n-2)}\left\{\frac{\alpha}{\left(\alpha b-a\left(\beta^{3}\right)(\alpha c-a \gamma)\right.} \cdot\left(\frac{a}{\alpha}\right)^{m+2}\right. \\
\left.\quad+\frac{\beta}{(\beta a-b a)(\beta c-b \gamma)} \cdot\left(\frac{b}{\beta}\right)^{m+2}+\frac{\gamma}{(\gamma a-c \alpha)(\gamma b-c \beta)} \cdot\left(\frac{c}{\gamma}\right)^{m+2}\right\} .
\end{gathered}
$$

Das analoge Resultat für das doppelte Integral ist:

$$
\begin{gathered}
\int_{0}^{\infty} d x \int_{0}^{\infty} \frac{(a x+b y)^{m}}{(\alpha x+\beta y+\mu)^{n}} d y \\
=\frac{1}{\mu^{n-m-2}} \frac{\Gamma(m+1) \Gamma(n-m-2)}{(n-1)}\left\{\frac{1}{\Gamma(n-1)}\left(\frac{a}{a \beta-\alpha b}\right)^{m+1}+\frac{1}{b \alpha-\beta a}\left(\frac{b}{\beta \sigma}\right)^{m+1}\right\} .
\end{gathered}
$$

Endlich werde noch angenommen, es sei:

$$
f(t)=e^{-t} \text {. }
$$

Unsere Formel liefert dann wieder drei einfache Integrale für das entsprechende dreifache Integral. Da aber nach einer allgemeinen Formel von

\section{Cauchy}

$$
\int_{0}^{\infty} d x \int_{0}^{\infty} d y \int_{0}^{\infty} \frac{e^{-(a x+b y+c z)}}{(\alpha x+\beta y+\gamma z+\mu)^{n}} d z=\frac{1}{\Gamma(n)} \int_{0}^{\infty} \frac{t^{n-1} e^{-\mu t} d t}{(\alpha t+a)(\beta t+b)(\gamma t+c)}
$$

ist, so ergiebt sich, durch Vergleichung beider Resultate und nach einigen Umformungen der resultirenden Gleichung, folgende bemerkenswerthe Relation:

$$
\int_{0}^{\infty} \frac{z^{n} e^{-k z} d z}{(\alpha z+a)(\beta z+b)(\gamma z+c)}=\frac{\Gamma(n+1)}{n(n-1) k^{n-2}}\left\{\begin{array}{c}
\frac{a^{n}}{(\alpha b-a \beta)(\alpha c-a \gamma)} \int_{0}^{\infty} \frac{c^{-k z} d z}{(\alpha z+a)^{n-1}} \\
+\frac{b^{n}}{(\beta a-b \alpha)(\beta c-b \gamma)} \int_{0}^{\infty} \frac{e^{-k z} d z}{(\beta z+b)^{n-1}} \\
+\frac{c^{n}}{(\gamma a-c \alpha)(\gamma b-c \beta)} \int_{0}^{\infty} \frac{e^{-k z} d z}{(\gamma z+c)^{n-1}}
\end{array}\right\}
$$

mittels welcher sich das Integral links, wenn $n$ eine positive ganze Zahl ist, 
1. Winclker, über die Reduction dreifacher Integrale auf Quadraturen.

insofern finden läfst, als

$$
\int_{0}^{\infty} \frac{e^{-k z} d z}{(\alpha z+u)^{n-1}} \text { etc. }
$$

durch partielle Integration auf Integral-Logarithmen zurückgeführt werden können.

15.

Eben so leicht wie in den bisherigen Fällen werden sich auch die Reductionsformeln herstellen lassen, wenn für $\boldsymbol{F}(x, y, z)$ einer der Ausdrücke $\cos (l x+\lambda) \cos (m y+\mu) \cos (n z+\nu), \quad \log (l x+\lambda) \log (m y+\mu) \log (n z+\nu)$,
$\cos (\alpha x+\beta y+\gamma z+\mu), \ldots \ldots$

gesetzt wird. Es finden sich dadurch jedesmal neue Resultate.

Sind die Grenzen des dreifachen Integrals unendlich, so lassen sich, den früheren Erörterungen zufolge, leicht allgemeine Gleichungen aufstellen, welche die meisten der bisherigen Ergebnisse als specielle Fälle enthalten.

So erhält man z. B., mit Berücksichtigung der Gleichung (A. S. 1.), sogleich :

$$
\begin{gathered}
\int_{0}^{\infty} d x \int_{0}^{\infty} d y \int_{0}^{\infty} f(a x+b y+c z) F(x, y, z) d z \\
=\frac{1}{c} \int_{1}^{\infty} f(t) d t \int_{0}^{\frac{t}{b}} d y \int_{0}^{\frac{t-b y}{a}} \mathrm{~F}(x, y) d x=\frac{1}{a} \int_{0}^{\infty} f(t) d t \int_{0}^{\frac{t}{c}} d z \int_{0}^{\frac{t-c z}{b}} \mathrm{~F}(y, z) d y \\
=\frac{1}{b} \int_{0}^{\infty} f(t) d t \int_{0}^{\frac{t}{a}} d x \int_{0}^{\frac{t-a x}{c}} \mathrm{~F}(x, z) d z .
\end{gathered}
$$

Ferner ist, wenn man

$$
\boldsymbol{F}\left(x, y, \frac{1}{c} \sqrt{ }\left(t-a x^{2}-b y^{2}\right)=\mathrm{F}(x, y)\right. \text { etc. }
$$

setzt:

$$
\begin{aligned}
& \int_{0}^{\infty} d x \int_{0}^{\infty} d y \int_{0}^{\infty} f\left(a x^{2}+b y^{2}+c z^{2}\right) H^{\prime}(x, y, z) d z \\
= & \frac{1}{2 \sqrt{ } c} \int_{0}^{\infty} f(t) d t \int_{0}^{\sqrt{ } \frac{t}{b}} d y \int_{0}^{\sqrt{ } \frac{t-b y^{2}}{a}} \frac{F(x, y) d x}{\sqrt{\left(t-a x^{2}-b y^{2}\right)}}=\text { etc. }
\end{aligned}
$$

Es wäre leicht, den hierbei zu Grunde liegenden Satz allgemein zu formuliren. Nur müssen, wie sich versteht, die Functionen $f$ und $\boldsymbol{F}$ oder, im Allgemeinen wenigstens, ihr Product ins Unendliche abnehmen, wenn die 
Veränderlichen ohne Ende wachsen. Ähnliche Formeln erhält man auch für doppelte Integrale.

16.

So viel mir bekannt, hat man sich mit der Reduction dreifacher Integrale, bei welchen die Function von swei getrennten Argumenten abhangt, bis jetzt nicht beschäftigt. Die Reduction ist in sehr vielen Fällen ebenfalls durch ein geometrisches Verfahren ausführbar und die Resultate übertreffen alle bisherigen an Allgemeinheit. Um den Gang in Kürze zu bezeichnen, seien $t$ und $\tau$ die zwei gegebenen Ausdrücke in $x, y, z$, von welchen die Function abhangt. Da sich für jeden Werth von $t$ und $\tau$ unendlich viele Werthe von $x, y, z$ angeben lassen, so lassen sich $t$ und $\tau$ als die Parameter zweier krummen Oberflächen betrachten, deren Durchschnitt im Allgemeinen eine Curve von doppelter Krümmung ist. Ändern sich nun jene Gröfsen um $d t$ und $d \tau$, so entstehen zwei weitere Flächen, welche mit den beiden früheren ein Element des Raums von unendlich kleinem Querschnitte einschliefsen, welches man, um seine Gestait zu bezeichnen, etwa ein gewundenes Prisma nennen könnte. Schneidet man dasselbe durch eine mit xy parallele Ebene, so ist der Schnitt ein kleines Parallelogranım, welches sich in seiner natürlichen Gestalt auf $x y$ projicirt und welches, mit $d z$ multiplicirt, das Volumen der unendlich kleinen Schicht jenes Prisma's giebt. Der Inhalt des Parallelogramms läfst sich allgemein ausdrücken, so dafs sich für das genannte Volumen

$$
\pm\left\{\left(\frac{d x}{d \tau}\right)\left(\frac{d y}{d t}\right)-\left(\frac{d x}{d t}\right)\left(\frac{d y}{d \tau}\right)\right\} d \tau d t d z
$$

findet. Um hieraus das prismatische Elemeni abzuleiten, ist zuerst nach : zu integriren, und zwar zwischen Grenzen, welche sich aus der Lage der Puncte ermitteln lassen, in welcher das Element die, durch die Grenzen des Integrals bestimmten Theile der Oberfläche des ganzen Raums trifft. Man integrire hierauf nach $t$, wobei $\tau$ constant bleibt, und dann schliefslich nach $\tau$, jedesmal innerhalb der Grenzen, zwischen welchen der zu berechnende Bestandtheil von den nämlichen Linicn oder Flächen eingeschlossen bleibt, so wird man die einzelnen Integrale erhalten, aus deren Summe das dreifache Integral besteht. Die Zahl dieser Theile, welche sich im Allgemeinen nicht weiter vereinigen lassen, beträgt im Allgemeinen wieder sieben.

Um diese Bemerkungen auf einen bestimmten, möglichst einfachen 
Fall anzuwenden, selzen wir zwei lineare Ausdrücke $t$ und $\tau$ und nehmen die Grenzen des Integrals als constant an.

Die sich schneidenden Flächen

$$
t=a x+b y+c z, \quad \tau=\alpha x+\beta y+\gamma z
$$

sind also Ebenen, und der zwischen je vier auf einander folgenden Ebenen dieser Art enthaltene Raumtheil ist ein gerades Prisma.

Wenn man nun, um sich hinsichtlich der Lage jener Ebenen auf einen ganz bestimmten Fall beziehen zu können, annimmt, es seien alle Coëfficienten positiv und es sei anfserdem:

$$
\frac{a}{\alpha}>\frac{b}{\beta}>\frac{c}{\gamma}
$$

so) hat eine unendlich dünne Schicht des Prisma's:

$$
\frac{\int(t, \tau)}{a \beta-\alpha b} d \tau d t d z
$$

also das Prisma selbst:

$$
\frac{f(t, \tau)}{u \beta-\alpha b}(z+\text { Const. }) d \tau d t
$$

zum Ausdrucke.

Das dreifache Integral kann in dem vorliegenden Falle nicht durch weniger als sieben Doppel-Integrale ausgedrückt werden, deren geometrische Darstellung man findet, wenn man, wie in (\$.2.) parallele Ebenen $(\tau)$ durch die daselbst angegebenen Eckpuncte des parallelepipedischen Raumes $(\xi, \eta, \zeta)$ legt.

17.

Die obigen Annahmen rücksichtlich der Coëfficienten haben zur Folge, dafs die Ebenen der beiden Systeme ( $t$ und $\tau$ ) die positiven Halb-Axen der $x, y, z$ schneiden und dafs eben so die Projection ihrer Durchschnittslinien auf $x y$ die beiden entsprechenden positiven Halb-Axen schneidet.

Die Bestimmung der Grenzen ist oben im Allgemeinen bezeichnet worden; sie ist etwas beschwerlich, ohne jedoch schwierig zu sein. Eine detailirte Ausführung derselben würde zu umständlich sein, während sich die Grenzen-Ausdrücke, bei Benutzung einer richtigen Figur fast ohne Weiteres hinschreiben lassen.

Indem wir also das Weitere übergehen und uns auf das Endresultat beschränken, möge nur noch die Bemerkung ihre Stelle finden, dafs sich das System von Werthen, wie es sich, unter Beibehaltung einer und derselben 
30 1. Winckler, über die Reduction dreifacher Integrale auf Quadraturen.

Aufeinanderfolge der Veränderlichen aus der Figur ergiebt, leicht in ein anderes transformiren läfst, wobei der Reihe nach jedesmal eine andere Veränderliche eliminirt ist, und wodurch die Theile des Integrals eine symmetrische Form bekommen. Der letztere Umstand ist um so mehr zu beachten, da die Symmetrie der Formeln, hier, wie in den früheren Fällen, das sicherste Mittel zur Prüfung der Rechnung abgiebt.

Setzt man wieder zur Abkürzung:

$$
\beta c-b \gamma=a_{1}, \quad \gamma a-c \alpha=b_{1}, \quad \alpha b-a \beta=c_{1},
$$

so findet man schliefslich folgende Gleichung:

$$
\begin{aligned}
& \int_{1}^{\xi} d x \int_{0}^{\eta} d y \int_{0}^{\zeta} f(a x+b y+c z, \alpha x+\beta y+\gamma z) d z \\
& =\int_{11}^{\alpha \xi} d \tau \int_{1}^{\frac{a}{\alpha} \tau} \frac{\alpha t-a \tau}{b_{1} c_{1}} f(t, \tau) d t+\int_{0}^{b \eta} d \tau \int_{0}^{\frac{b}{b} \tau} \frac{\beta t-b \tau}{a_{1} c_{1}} f(t, \tau) d t \\
& +\int_{0}^{\gamma \zeta} d \tau \int_{0}^{\frac{c}{\gamma} \tau} \frac{\gamma t-c \tau}{a_{1} b_{1}} f(t, \tau) d t \\
& -\int_{\alpha \xi}^{\alpha \dot{\xi}+b \eta} d \tau \int_{0}^{\frac{b \tau-c_{1} \xi}{\beta}} \frac{\beta t-b \tau+c_{1} \xi}{a_{1} c_{1}} f(t, \tau) d t-\int_{\beta \eta}^{\alpha \xi+\beta \eta} d \tau \int_{0}^{\frac{a \tau+c_{1} \eta}{\alpha}} \frac{\alpha t-a \tau-c_{1} \eta}{b_{1} c_{1}} f(t, \tau) d t \\
& -\int_{\beta \eta}^{\beta \eta \eta+\gamma \zeta} d \tau \int_{0}^{\frac{c \tau-a_{1} \eta}{\gamma}} \frac{\gamma t-c \tau+a_{1} \eta}{a_{1} b_{1}} f(t, \tau) d t-\int_{\gamma \zeta}^{\beta \eta+\gamma_{\zeta}^{*}} d \tau \int_{0}^{\frac{b \tau+a_{1} \zeta}{\beta}} \frac{\beta t-b \tau-a_{1} \zeta}{a_{1} c_{1}} f(t, \tau) d t \\
& -\int_{\gamma \zeta}^{\alpha \xi+\gamma \zeta} d \tau \int_{0}^{\frac{a \tau-b_{1} \zeta}{\alpha}} \frac{\alpha t-a \tau+b_{1} \xi}{b_{1} c_{1}} f(t, \tau) d t-\int_{\alpha \xi}^{\alpha \xi+\gamma \zeta} d \tau \int_{0}^{\frac{c \tau+b_{1} \xi}{\gamma}} \frac{\gamma t-c \tau-b_{1} \xi}{a_{1} b_{1}} f(t, \tau) d t \\
& +\int_{\alpha \xi+\beta \eta}^{\alpha \xi+\beta \eta+\gamma \zeta} d \tau \int_{0}^{\frac{c \tau+b_{1} \xi-a_{1} \eta}{\gamma}} \frac{\gamma t-c \tau-b_{1} \xi+a_{1} \eta}{a_{1} \nu_{1}} f(t, \tau) d t \\
& +\int_{\beta \eta+\gamma \zeta}^{\alpha \xi+\beta \eta+\gamma \zeta} d \tau \int_{0}^{\frac{a \tau+c_{1} \eta-b_{1} \zeta}{\alpha}} \frac{\alpha t-a \tau-c_{1} \eta+b_{1} \zeta}{b_{1} c_{1}} f(t, \tau) d t \\
& +\int_{\alpha \xi+\gamma \zeta}^{\alpha \xi+\beta \eta+\gamma \zeta} d \tau \int_{0}^{\frac{b \tau+a_{1} \zeta-c_{1} \xi}{\beta}} \frac{\beta t-b \tau-a_{1} \xi+c_{1} \xi}{a_{1} c_{1}} f(t, \tau) d t .
\end{aligned}
$$

Wenn $f(t, \tau)$ so schnell abnimmt, dafs für $\xi=\eta=\zeta=\infty$ die Integrale, bei welchen diese Gröfsen in den untern und obern Grenzen zugleich vorkommen, der Null sich nähern, so reducirt sich das dreifache Integral 
1. Winclkler, über die Reduction dreifacher Integrale auf Quadraturen. 31 auf die drei ersten Doppel-Integrale und man erhält:

$$
\begin{gathered}
\int_{0}^{\infty} d x \int_{0}^{\infty} d y \int_{0}^{\infty} f(a x+b y+c z, \alpha x+\beta y+\gamma z) d z \\
=\int_{0}^{\infty} d \tau \int_{0}^{\frac{a}{\alpha} \tau} \frac{(a \tau-\alpha t) f(t, \tau)}{\left(\alpha b-a \beta^{\prime}\right)(\alpha c-a \gamma)} d t+\int_{0}^{\infty} d \tau \int_{1}^{\frac{b}{\beta} \tau} \frac{(b \tau-\beta t) f(t, \tau)}{(\beta a-b \alpha)(\beta c-b \gamma)} d t \\
+\int_{0}^{\infty} d \tau \int_{0}^{\frac{c}{\gamma} \tau} \frac{(c \tau-\gamma t) f(t, \tau)}{(\gamma a-c \alpha)(\gamma b-c \beta)} d t .
\end{gathered}
$$

Formeln dieser Art lassen sich auch noch für viele andere Formen von $t$ und $\tau$ finden. Sofern es sich aber um eine explicite Darstellung der Reductionsformeln handelt, werden die Resultate so weitläuftig, dafs sie sich hier zur Mittheilung kaum mehr eignen.

Zum Schlusse noch die Bemerkung, dafs frühere Andeutungen, so wie die Resultate selbst, sehr leicht auf die Bildung der entsprechenden $4,5, \ldots n$ fachen Integrale führen.

Brünn, 3 August 1853. 